\title{
Real-Time MIMO Channel Sounder for Emulation of Distributed Ultrawideband Systems
}

\author{
Rudolf Zetik, Martin Kmec, Jürgen Sachs, and Reiner S. Thomä \\ Ilmenau University of Technology, PF 100565, 98684 Ilmenau, Germany \\ Correspondence should be addressed to Rudolf Zetik; rudolf.zetik@tu-ilmenau.de
}

Received 20 March 2014; Accepted 14 August 2014; Published 15 September 2014

Academic Editor: Christoph F. Mecklenbräuker

Copyright ( 2014 Rudolf Zetik et al. This is an open access article distributed under the Creative Commons Attribution License, which permits unrestricted use, distribution, and reproduction in any medium, provided the original work is properly cited.

\begin{abstract}
This paper introduces an ultrawideband (UWB) channel sounding system. Its novel architecture allows real-time measurements of multiple time-variant radio propagation channels in different ultrawide frequency bands. Its architecture allows emulation of multiuser systems, sensor networks, localization systems, and distributed MIMO radar systems. The sounder uses a maximum length binary sequence (MLBS) excitation signal and correlation processing in the receiver. Its synchronous multichannel operation is supported by excellent timing stability and low power consumption of miniature size modules based upon custom integrated $\mathrm{SiGe}$ circuits. The paper describes the architecture, design, calibration, basic parameters, and application examples of the sounding system.
\end{abstract}

\section{Introduction}

UWB wireless systems are often discussed as a prospective technology for short range indoor communication and radar sensing applications [1-3]. UWB systems benefit from the large frequency band which usually extends over several $\mathrm{GHz}$. Such a frequency range results in an excellent multipath resistance and enables precise localization. This makes UWB radio one of the most promising technologies that realizes indoor communication systems with integrated localization and tracking application, location-aware sensor networks, through-wall radars, and so forth.

The proper design of such communication and sensing UWB systems requires the knowledge of propagation characteristics that are application specific radio environments. This information can be obtained from the time-variant channel impulse response function (CIRF) which describes the end-to-end propagation channel. CIRF includes information about the delay spread, the coherence bandwidth, time-varying multipath weights, and others. Depending on antenna characteristics and spatial antenna arrangements even polarization and spatial/directional characteristics of the channel can be estimated from CIRFs.

There has been a great deal of effort directed to investigate the radio channel characteristics from CIRF measured by channel sounders in real environments [4,5]. Although those existing real-time sounding systems are often called "broadband," they do not meet extreme bandwidth requirements of the UWB technology. Their bandwidth is typically limited to $100 \mathrm{MHz}-240 \mathrm{MHz}$. This is by far not enough to investigate UWB propagation phenomena in frequency bands that extend over several $\mathrm{GHz}$ that were deregulated for the license free indoor usage [6-8]. Examples are given by UWB bands that were deregulated for communication applications, for example, in Europe from $6 \mathrm{GHz}$ to $8.5 \mathrm{GHz}$, or in USA from $3.1 \mathrm{GHz}$ to $10.6 \mathrm{GHz}$ (the well-known Federal Communication Commission (FCC) mask). These deregulated bands widely open doors for mass market in the field of UWB communication. However, for good material penetration the lower frequency limits are too high. If UWB sensors shall "look" through walls then especially the low frequencies are very important. These requirements were also reflected by decisions made by regulatory bodies, for example, in Europe by Electronic Communications Committee radiation limits for selected UWB applications that differ from the generic limits imposed on UWB communication devices. An overview of deregulated bands can be found, for example, in [9].

The existing channel and propagation studies cannot be simply scaled up to these frequency bands because of 
TABLE 1: Comparison of UWB channel sounding architectures.

\begin{tabular}{|c|c|c|c|c|c|}
\hline Principle & $\begin{array}{l}\text { MLBS generator } \\
\text { and RTO [19] }\end{array}$ & $\begin{array}{c}\text { Sliding correlator } \\
{[20]}\end{array}$ & AWG and RTO [17] & Conventional VNA & $\begin{array}{l}\text { Proposed MLBS } \\
\text { based prototype }\end{array}$ \\
\hline Excitation signal & $\begin{array}{c}\text { PNS } \\
1023 \text { chips }\end{array}$ & $\begin{array}{c}\text { PNS } \\
131017 \text { chips }\end{array}$ & $\begin{array}{c}\text { PNS } \\
2047 \text { chips }\end{array}$ & Stepped frequency & $\begin{array}{l}\text { PNS } \\
4095\end{array}$ \\
\hline Channels & 1 & 1 & 1 & $\sim 2$ & $2 \mathrm{Tx} 4 \mathrm{Rx}$ \\
\hline Frequency band & $3.6-6.0 \mathrm{GHz}$ & $3.5-4.7 \mathrm{GHz}$ & $2-12 \mathrm{GHz}$ & $\begin{array}{l}\text { Typically } \\
0-26 \mathrm{GHz}\end{array}$ & $\begin{array}{c}\mathrm{DC}-3.5 \mathrm{GHz} \\
3.5-10.5 \mathrm{GHz} \\
59.5-66.5 \mathrm{GHz} \\
\end{array}$ \\
\hline Measurement rate & Not available (NA) & $0.077 \mathrm{~Hz}$ & $2.5 \mathrm{~Hz}$ & $\begin{array}{c}\text { typ. less than } \\
2 \mathrm{~Hz} @ 40 \mathrm{~dB} \\
0.2 \mathrm{~Hz} @ 90 \mathrm{~dB} \\
\end{array}$ & $\begin{array}{c}100 \mathrm{~Hz} \\
\text { in 1Tx-4Rx } \\
\text { configuration } \\
\end{array}$ \\
\hline $\begin{array}{l}\text { Emulation of distributed } \\
\text { systems }\end{array}$ & $\begin{array}{c}\text { Long } \\
\text { synchrocable }\end{array}$ & Wireless synchro & $\begin{array}{c}\text { Long } \\
\text { antenna cables }\end{array}$ & $\begin{array}{c}\text { Long } \\
\text { antenna cables }\end{array}$ & $\begin{array}{c}\text { Long synchro, } \\
\text { or antenna cables, or } \\
\text { wireless }\end{array}$ \\
\hline AGC & No & No & No & No & Yes $0-40 \mathrm{~dB}$ \\
\hline $\begin{array}{l}\text { Instantaneous dynamic } \\
\text { range }\end{array}$ & NA & $34 \mathrm{~dB}$ & $\begin{array}{c}\text { NA } \\
\text { usually }<60 \mathrm{~dB}\end{array}$ & $\begin{array}{l}\text { Typically } \\
<120 \mathrm{~dB}\end{array}$ & $\begin{array}{l}67 \mathrm{~dB} \text { for wired } \\
\text { synchronization }\end{array}$ \\
\hline
\end{tabular}

the strong frequency-selectivity which is due to the frequency varying structural and material dependent scattering and transmission characteristics [10]. Moreover, it is well known that there are several advantages to have the sounder bandwidth wider than the respective application system. Larger bandwidth gives better insight into the multipath structure of the channel. This allows more accurate reproduction of the channel statistics by models that are deduced from measured data. Therefore, UWB channel sounders with a frequency band of several $\mathrm{GHz}$ are inevitable for the proper design of UWB communication and sensing systems.

The majority of UWB channel measurements reported in the open literature are carried out by general purpose vector network analyzers (VNA) [10-16], or by arbitrary waveform generator (AWG) and real-time oscilloscopes (RTO) [17, 18]. VNA sounding that uses mainly sine-wave excitation easily allows enough bandwidth by slow frequency sweeping. Therefore its application is restricted to measurements where transmitters and receivers are kept static and the scenario is time invariant. The huge measurement time prohibits sounding in realistic scenarios with moving transmitters, receivers, objects, or people. For time-variant scenarios, other hardware architectures are necessary. Some of them are already known, especially in the field of UWB radars. They mainly rely on application of short impulses or spread spectrum correlationtechniques. However, their application in the field of the real-time UWB channel sounding is often subjected to a number of constraints such as insufficient measurement rate, a bandwidth limited to just one frequency band, too complex electronics, high susceptibility to jitter, large drift, or others. For example, the UWB sounding presented in $[19,20]$ was inspired by the UWB radar sensor architecture based on the spread spectrum correlation techniques and impulse compression of pseudonoise sequences (PNS). However, these sounding architectures offer only small bandwidth; they do not allow MIMO measurements or the measurement rate is too low for the real-time analysis of time variant channels.

In this paper, we provide a compact description of a UWB channel sounder, which can operate in real-time.
Its architecture supports measurement rate up to several thousand CIRFs per second. This measurement rate allows sounding in majority of time-variant indoor and outdoor scenarios with moving objects and people. It has multichannel architecture for sounding in MIMO-systems. Its modular design supports emulation of systems with collocated and even distributed antennas.

The prototype of this sounder was developed in the framework of European integrated projects PULSERS I and II (Pervasive Ultrawideband Low Spectral Energy Radio Systems) and EUWB (coExisting short range radio by advanced ultrawideBand radio technology) [21]. The very first published realization of the sounder was restricted to the baseband operation up to $3.5 \mathrm{GHz}$ [22]. Later, the sounder became capable of passband operation from $3.5 \mathrm{GHz}$ to $10.5 \mathrm{GHz}$ [23]. This paper describes the recent stage in the development of this real-time sounder that allows multichannel UWB measurements in three different frequency bands: DC3.5 GHz, 3.5 GHz-10.5 GHz, and $59.5 \mathrm{GHz}-66.5 \mathrm{GHz}$. Within this paper we will refer to these bands as: the baseband, the FCC band, and the $60 \mathrm{GHz}$ band, respectively. Note that the notation of the "FCC band" reflects the proximity of the $3.5 \mathrm{GHz}-10.5 \mathrm{GHz}$ frequency band to the frequencies given by the FCC mask but not the exact match to it. The sounder is equipped with automatic gain control (AGC) and it has modular design. This is beneficial for the emulation of distributed systems. The main differences of the described sounder to the most significant existing channel sounding approaches are highlighted in Table 1.

The paper starts with the discussion about basic requirements imposed on real-time UWB sounders. Considering these requirements, a sounder architecture and its design based upon custom integrated analog and digital SiGecircuits is introduced. Afterwards, the paper describes the calibration procedure and sounders' basic parameters. Finally, an application of the sounder is illustrated by a selected measurement example.

Since the paper provides an extended description of the sounder and a number of related topics we want to 
highlight the main novel contributions of the article that can be summarized as

(i) compact description of the sounder,

(ii) analysis of the measurement rate for real-time channel sounding in time-variant scenarios,

(iii) the sounder calibration, especially the IQ mismatch (imbalance) calibration,

(iv) application example in distributed systems without any synchronization between the transmitter and the receiver, which demonstrates a new direction in the UWB channel sounding for emulation and evaluation of sensor networks.

\section{Basic Requirements}

Apart from its extreme bandwidth of several GHz, the realtime UWB channel sounder has to offer a high measurement rate, full MIMO capability, AGC, and modular and scalable design.

A high measurement rate is important requirement on a real-time channel sounder which probes the radio channel by a periodic excitation signal and provides estimates of a 2 dimensional CIRF $h(\tau, t)$. Here, the time delay axis $\tau$ is related to the time of flight of electromagnetic (EM) waves and the measurement time axis $t$ is related to the time evolution of the radio channel. Sampling of the time delay axis $\tau$ is given by the sampling rate of the ADC in the sounder's receiver. Measurement rate of the sounder determines the sampling rate of $\operatorname{CIRF} h(\tau, t)$ along the axis $t$. The measurement rate of UWB measurement devices is usually at most several hundred CIRFs per second with current technological capabilities and affordable costs. Therefore, the time measurement axis $t$ is often referred to as the slow-time axis and the delay time axis $\tau$ with the equivalent sampling of tens of $\mathrm{GHz}$ is referred to as the fast-time axis. The measurement rate must be high enough to match the time variance of the channel. If the measurement rate is too low, the time evolution of the CIRF cannot be completely recovered from the measured data. Since the time variance of the channel is mostly caused by moving objects or moving antennas of the sounder, the measurement rate determines the maximum relative radial velocity of objects and antennas that is allowed in the inspected scenario or vice versa.

The MIMO capability is necessary for emulating multiantenna and multiuser communication systems, for distributed sensor networks, distributed MIMO radar, for dual polarimetric measurements, and so forth. Established wideband MIMO sounders [24-28] usually rely on a single channel RF transmitter and receiver architecture. In this case, MIMO antennas are accessed by RF switches. However, antenna switching is more suitable for collocated antenna arrays than for distributed sounding systems addressed in this paper. Moreover, simple scaling of the one channel architecture does not meet typical UWB requirements like the huge bandwidth and the high measurement rate. Therefore, the proposed UWB sounder is based on a true parallel multichannel architecture.
AGC is a prerequisite to efficiently use the sounder's dynamic range in real-time measurements, which may undergo a large variation of the input signal power level. This variation may reach even hundred decibels and would decrease quality of real-time measurement by too low signalto-noise ratio (SNR) or operation of sounder's input circuitry in the saturation. AGC is an adaptive system that can be found in many electronic devices. The output signal level is fed back to adjust the gain of input amplifiers to an appropriate level. In general most of the known channel sounder systems allow AGC switching between consecutively measured CIRFs.

The modular and scalable design should offer a high degree of hardware configuration flexibility to allow adaptation of the system to actual requirements of individual users.

\section{Measurement Rate}

Since the measurement rate is critical for sounding of time variant channels we analyze dependency between the measurement rate and the maximum relative radial speed of objects within the measurement scenario. In order to derive the basic dependency let us assume the following simplified scenario. One point-like scatterer moves with a constant velocity $v$ in the radial direction towards a monostatic antenna. A real-time channel sounder probes the radio channel by an UWB excitation signal $e(\tau)$ which has the bandwidth $B$ at the carrier frequency $f_{c}$ and it is transmitted with the repetition rate equal to $1 / T_{\mathrm{Tx}}$. Such an excitation signal can be described in the time domain as

$$
e(\tau)=\operatorname{Sinc}[\tau B] e^{-j 2 \pi f_{c} \tau} \otimes \sum_{i=0}^{\infty} \delta\left(\tau-i T_{\mathrm{Tx}}\right),
$$

where Sinc represents the normalized Sinc function, $\delta(\tau)$ is the Dirac pulse, and $\otimes$ is the convolution. In the ideal case, the signal which is scattered from the moving object and received by the sounder at time $t$ is described for $\tau<T_{\mathrm{Tx}}$ by

$r(\tau, t)=P(t) \operatorname{Sinc}\left[\left(\tau-\frac{2\left(d_{1}-v t\right)}{c}\right) B\right] e^{-j 2 \pi f_{c}\left(\tau-2\left(d_{1}-v t\right) / c\right)}$,

where $c$ is the speed of light, $d_{1}$ is the distance between the object and the antenna at time $t_{1}$ and $P(t)$ includes the path loss caused by the channel, antennas and so on. The received signal $r(\tau, t)$ must be properly sampled along both axes in order to allow the information about the time-variant radio channel to be completely recoverable from the sampled signal. In order to avoid sampling of the signal at the carrier frequency $f_{c}$, the sounder usually uses IQ down-conversion, which shifts received signals to the baseband. The complex valued down-converted signal for $\tau<T_{\mathrm{Tx}}$ and for the assumed scenario is given by

$$
r(\tau, t)=P(t) \operatorname{Sinc}\left[\left(\tau-\frac{2\left(d_{1}-v t\right)}{c}\right) B\right] e^{j 2 \pi f_{c}\left(2\left(d_{1}-v t\right) / c\right)} .
$$

The Fourier transform of (3) with respect to $\tau$ for a certain measurement time $t$ is

$$
R\left(f_{\tau}, t\right)=K_{1}(t) \operatorname{Rect}\left[B^{-1} f_{\tau}\right],
$$


where $f_{\tau}$ is the frequency axis related to the short-time axis, $K_{1}(t)$ is a complex constant which depends on the slow-time, and Rect is the rectangular function. According to characteristics of the rectangular function and (4) the sampling frequency along the fast-time axis must be at least the bandwidth of the excitation signal $B$. Similarly, the Fourier transform of (3) with respect to $t$ for a certain time delay $\tau$ under assumption of slowly time varying $P(t)$, in which time-dependency is neglected, results in

$$
R\left(f_{t}, \tau\right)=K_{2}(\tau) \operatorname{Rect}\left[\left(2 \frac{v}{c} B\right)^{-1}\left(f_{t}-2 \frac{v}{c} f_{c}\right)\right],
$$

where $f_{t}$ is the frequency axis related to the slow-time axis, $K_{2}(\tau)$ is a complex constant which depends on the fasttime. This equation represents the "spectrum" of the twodimensional CIRF along the slow-time axis $t$. It is apparent that the spectral components cover the "bandwidth" $2 v B / c$ that is "modulated" at the frequency $2 v f_{c} / c$. Thus, the minimum sampling frequency along the slow-time axis $t$ which is equal to the measurement rate $M$ is

$$
M \geq 2 \frac{v}{c}\left(B+f_{c}\right) .
$$

This gives the basic dependency between the measurement rate $M$ and the maximum relative radial speed $v$ of objects. Note that (6) was derived only for objects which move at a constant speed without any acceleration and under the assumption that the measurement time of time-variant CIRF is short enough so that the radio channel can be treated as time invariant within this measurement time. This is naturally not valid for realistic environments. In this case, the measurement rate must be even higher than the rate given by (6). A more detailed discussion on this topic is beyond the scope of this paper. However, (6) gives the reader at least a feeling about the necessary measurement rate or the maximum velocity of objects for real-time channel sounding in time-variant scenarios. To give an example let us assume a sounder is capable of measurements in 2 different bands. The first band is the FCC band that extends from $3.5 \mathrm{GHz}$ to $10.5 \mathrm{GHz}$. The second band is the $60 \mathrm{GHz}$ band from $59.5 \mathrm{GHz}$ to $66.5 \mathrm{GHz}$. Both bands have the same bandwidth of $7 \mathrm{GHz}$ but their carrier frequencies are different. If the radio channel is to be measured and analyzed in an office scenario with people that move mostly with, for example, $1 \mathrm{~m} / \mathrm{s}$ the minimum measurement rate according to (6) is for the FCC band about 93 complex valued measurements per second and for the $60 \mathrm{GHz}$ it is about 446 complex valued measurements per second. This value shows that the measurement rate must not be underestimated for realtime channel sounding in realistic time-variant scenarios especially at higher frequencies.

\section{Channel Sounder Design}

4.1. Excitation Signal. The key to design a powerful UWB sounder is the selection of an appropriate excitation signal. Short pulses and pseudonoise sequences known in directsequence spread spectrum systems are usually applied for time domain sounding. We choose a maximal length binary sequence (MLBS) which autocorrelation properties make it suitable for propagation channel sounding [29].

The advantage of MLBS is that they can easily be generated with large bandwidth by a digital shift register which is clocked by a stable RF-oscillator. Their waveform is beneficial with respect to the power distribution. MLBS have uniformly distributed power over time. This maximizes the energy of one MLBS period while keeping low peak voltages. For example, a short Gauss pulse requires about 200 times (for $B T_{\mathrm{Tx}}=1000$ ) larger amplitude than the MLBS if the energy, the bandwidth $B$, and the CIRF duration $T_{T x}$ have to be the same. The small MLBS amplitudes allow realization of the MLBS generator in low voltage integrated circuit technology like SiGe. This supports extremely fast digital switching that helps to meet the demanding requirements on bandwidth, low jitter and high SNR. Moreover, because of the binary signal waveform, cheap nonlinear power amplifiers are sufficient if increased output power is needed.

Further advantage of MLBS signals is their high correlation gain and an almost ideal compressed triangular correlation function. The spectral shape of the ideal MLBS signal follows a (sinc) $)^{2}$ function. This means that almost $80 \%$ of the energy is concentrated within the band from DC to half the clock rate. Thus, the same RF-clock which is used for the MLBS generation can be also used to control the sampling circuitry at the receiver side. In order to remove the residual $20 \%$ of the energy concentrated above half the clock rate an antialiasing filter is needed. However, it slightly impairs beneficial features of MLBS especially its correlation function. It is advantageous to use it at the receiver side. In this case, it removes even disturbing signals that do not originate from the sounder.

Another advantage of MLBS signals is their periodicity. It allows signal processing in the frequency domain without leakage effects that occur if frequency components are not harmonic with the FFT fundamental functions and result in the signal energy smearing over a wide frequency range. Another benefit of periodical MLBS signals is the cost effective periodic subsampling for signal recording. A certain degree of subsampling is allowed without data loss because of the limited time variation of the channel. However, it must be taken into account that the subsampling negatively influences the SNR, the efficiency, and the measurement rate. It was shown that the measurement rate is bounded to the time variation of the radio channel. That is why the subsampling is a certain tradeoff between real-time capability of the sounder, its SNR, efficiency, and overall costs.

4.2. Basic Architecture of the Sounder. The architecture of the proposed UWB sounder is conceptually based on a baseband MLBS radar chipset [30-34]. The sounder architecture generally consists of two parts - the baseband and the passband part (FCC and $60 \mathrm{GHz}$ band). The baseband part is designed for the channel sounding which frequency range varies from close to zero Hertz to a certain frequency which is given by half the system clock $f_{s}$ that drives the MLBS generator. The passband part is used to extend the baseband part and to shift the original baseband MLBS to higher frequencies by 


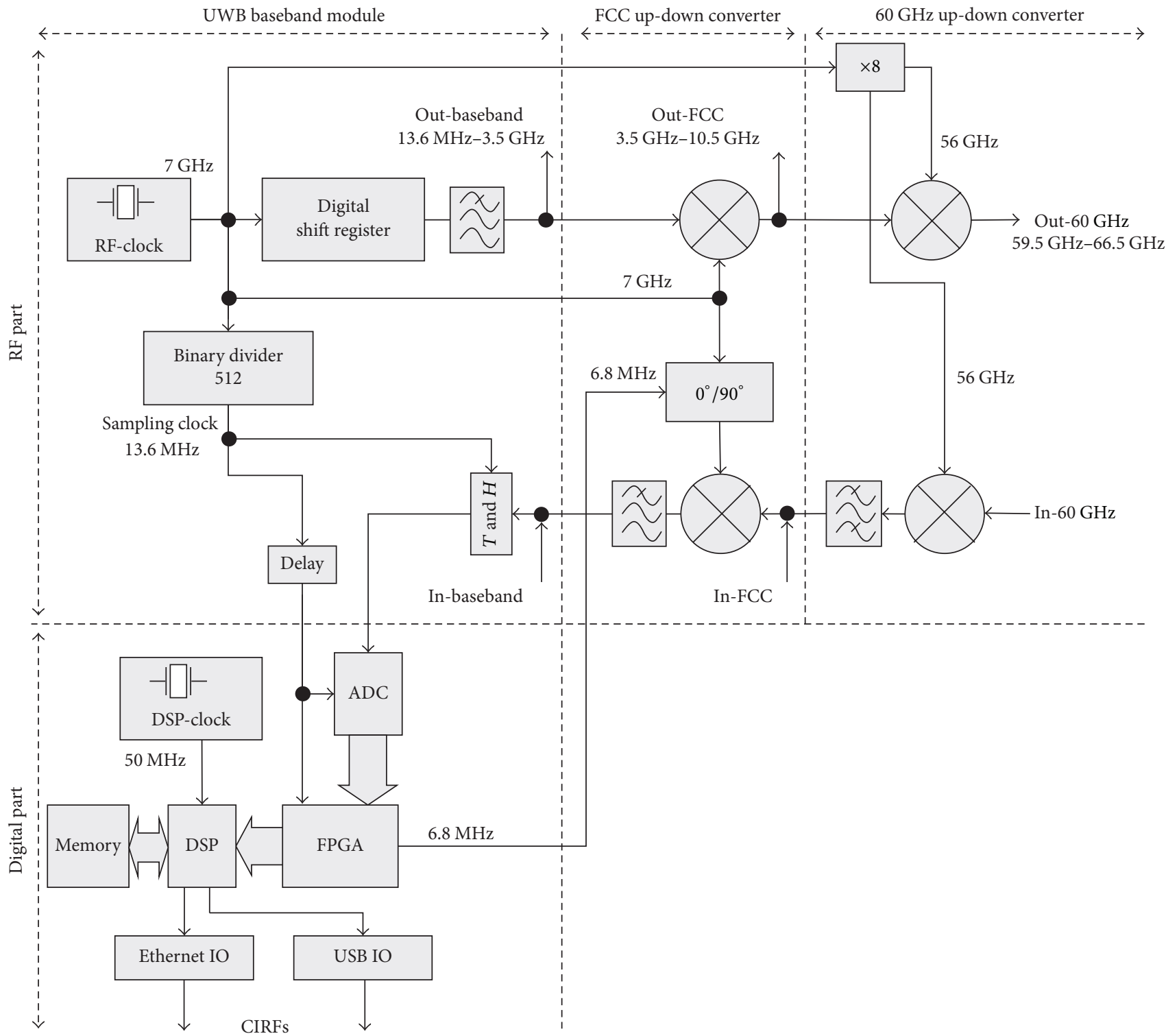

FIGURE 1: Architecture of the UWB channel sounder.

mixing it at a suitable carrier frequency $f_{c}$. This results in the excitation signal which spans a frequency band from $f_{c}-f_{s} / 2$ to $f_{c}+f_{s} / 2$ which is two times larger than the baseband frequency band. In order to avoid sampling of the signal at the carrier frequency $f_{c}$, the sounder uses IQ down-conversion, which shifts measured signals back to the baseband (see (3) and the related description). This results in a complex valued CIRF which has bandwidth twice as large as is the bandwidth of the real valued CIRF obtained by the baseband sounding. Therefore, passband sounding usually requires two receiving baseband channels with the bandwidth $f_{s} / 2$ to measure the real valued $I$ - and $Q$-parts of the complex valued passband CIRF.

Figure 1 shows basic (one channel) architecture of the proposed real-time UWB sounder. The baseband excitation signal is the MLBS which is generated by a high speed digital shift register. The shift register is driven by an RF-clock generator that works at the frequency $f_{s}$. Thus, the excitation signal covers baseband frequencies up to $f_{s} / 2$. On the receiver side of the baseband part, the subsampling clock is generated by a simple binary divider which allows very stable operation.

The output signal of the binary divider directly drives the track and hold circuit $(\mathrm{T} \& \mathrm{H})$ and the $\mathrm{AD}$-converter (ADC). If the order of the shift register and the order of the binary divider (number of flip-flops) are identical, the subsampling approach is known as the sequential sampling. If the order of the shift register is higher than the order of the binary divider, the subsampling approach is known as the interleaved sampling which takes more than one sample within one period. The order of data samples is scrambled, but this can be simply removed. The principle of interleaved sampling allows varying the sampling rate by keeping the sensor concept. Thereby, one can reduce the sampling rate in favor of reduced power consumption and 
device costs or it can also be increased to improve the receiver efficiency depending on the development state of high speed electronics.

In order to increase SNR of measured impulse responses, data can be averaged at the digital signal processor (DSP) of the digital module. However, this decreases the measurement rate of the sounder. Therefore, the number of averages as well as the subsampling rate should be chosen properly so that the measurement rate matches the time variance of the sounding scenario and (6) is kept valid. The digital module contains DSP and field programmable gate array (FPGA) for impulse compression, averaging, and communication control and for eventual implementation of application specific algorithms.

For the FCC band, the baseband RF-front-end is extended with an up-down frequency converter. The up-convertor shifts the baseband MLBS to higher frequencies by a double sided mixing. The original MLBS is mixed with a suitable carrier signal. For simplicity and most stable synchronous operation we use the RF-clock frequency $f_{s}$ as the carrier. This implicates that the main part of the excitation energy is concentrated in the frequency band from $f_{s}-f_{s} / 2$ to $f_{s}+f_{s} / 2$.

In order to approximately match the majority of frequency bands that were deregulated for the license free indoor usage, the clock rate $f_{s}$ of our sounder was fixed to $7 \mathrm{GHz}$. This resulted in the baseband operation up to $3.5 \mathrm{GHz}$ and the passband operation from $3.5 \mathrm{GHz}$ to $10.5 \mathrm{GHz}$ which approximately covers the FCC band and almost all other frequency bands as well.

On the receiver side of the "FCC part," the captured passband signal is IQ-down-converted to the complex valued baseband signal. The classical parallel IQ-demodulator concept was abandoned because of expected problems with the IQ imbalance. Instead, we realized a novel sequential IQ-demodulator (see Figure 1). Here, the phase of the LO (local oscillator) signal is sequentially switched between $0^{\circ}$ and $90^{\circ}$ after the whole measurement of one $I$ - (or Q-part) is completed. The advantage of this approach is twofold. It decreases the number of channels for the IQ-demodulation and simplifies the calibration. However, the prize for it is the two times decreased measurement rate.

For the $60 \mathrm{GHz}$ band, the FCC front-end is extended with an additional up-down frequency converter. This updown converter uses LO signal of $56 \mathrm{GHz}$. This frequency is obtained by $8 \mathrm{x}$ multiplication of the RF-clock frequency $f_{s}$. The mixing at this carrier frequency results in the excitation signal which spans a frequency band from $59.5 \mathrm{GHz}$ to $66.5 \mathrm{GHz}$. More details about the design and application of the $60 \mathrm{GHz}$ front-ends can be found in [35-39].

4.3. Data Processing. The sounder continuously generates periodical MLBS excitation signal $e(\tau)=e\left(\tau+T_{\mathrm{Tx}}\right)$. Its period is $T_{\mathrm{Tx}}=M T_{S}$, where $T_{S}=1 / f_{s}$ is the duration of one MLBS chip which is given by the system clock $f_{s}$ and $M=2^{N}-1$ is the number of chips within one MLBS period which is determined by the number of flipflops $N$ in the digital shift register. The excitation signal is influenced by the radio channel before it is received by the receiving antenna and sampled by the sounder. Due to the subsampling and synchronous averaging of the received signal the measurement time for one period of the MLBS signal is $T_{\mathrm{Rx}}=T_{\mathrm{Tx}} S A$, where $S$ is the subsampling factor and $A$ is the number of averages. During this measurement time the CIRF must be time-invariant with just negligible time variations that do not significantly disturb the measurement process. In this case, the sampled signal captured during the measurement time $T_{\mathrm{Rx}}$ can be described by the cyclic discrete convolution with added measurement noise $n_{T_{\mathrm{Rx}}}\left(m T_{S}\right)$ as

$$
r_{T_{\mathrm{RX}}}\left(m T_{S}\right)=\sum_{i=0}^{M-1} h_{T_{\mathrm{Rx}}}\left(i T_{S}\right) e\left(m T_{S}-i T_{S}\right)+n_{T_{\mathrm{Rx}}}\left(m T_{S}\right),
$$

if the duration of the impulse response $h_{T_{\mathrm{px}}}\left(m T_{S}\right)$ is shorter than one period of the excitation signal $T_{\mathrm{Tx}}$. Since the realtime channel sounder periodically captures the received signal, (7) can be reformulated to stress two dimensions (the fast time axis and the slow time axis) of the time variant measured signal $r\left(m T_{S}, n T_{\mathrm{Rx}}\right)$ and the impulse response $h\left(m T_{S}, n T_{\mathrm{Rx}}\right)$ which evolves in slow time axis $n T_{\mathrm{Rx}}$

$$
\begin{aligned}
& r\left(m T_{S}, n T_{\mathrm{Rx}}\right) \\
& \quad=\sum_{i=0}^{M-1} h\left(i T_{S}, n T_{\mathrm{Rx}}\right) e\left(m T_{S}-i T_{S}\right)+n\left(m T_{S}, n T_{\mathrm{Rx}}\right) .
\end{aligned}
$$

From the system identification theory, the impulse response $h\left(m T_{S}, n T_{\mathrm{Rx}}\right)$ can be estimated by applying WienerHopf theory [40]. The best approximation of (8) in the least squares sense is obtained by minimizing the squared error

$$
\begin{aligned}
& \operatorname{se}\left(n T_{\mathrm{Rx}}\right) \\
& =\frac{1}{2} \sum_{i=0}^{M-1}\left(r\left(i T_{S}, n T_{\mathrm{Rx}}\right)-\sum_{j=0}^{M-1} h\left(j T_{S}, n T_{\mathrm{Rx}}\right) e\left(i T_{S}-j T_{S}\right)\right)^{2}
\end{aligned}
$$

relative to $h\left(m T_{S}, n T_{\mathrm{RX}}\right)$. "The minimum is given by a set of equations defined by

$$
\frac{\partial \mathrm{se}\left(n T_{\mathrm{RX}}\right)}{\partial h\left(m T_{S}, n T_{\mathrm{RX}}\right)}=0,
$$

which results in the famous Wiener-Hopf equations

$$
C^{e r}\left(m T_{S}, n T_{\mathrm{Rx}}\right)=\sum_{i=0}^{M-1} h\left(i T_{S}, n T_{\mathrm{Rx}}\right) C^{e e}\left(m T_{S}-i T_{S}\right),
$$

where $C^{e r}$ is the cross-correlation of the ideal MLBS with one period of the received signal measured at time $n T_{\mathrm{Rx}}$

$$
C^{e r}\left(m T_{S}, n T_{\mathrm{Rx}}\right)=\sum_{i} r\left(i T_{S}, n T_{\mathrm{Rx}}\right) e\left(i T_{S}+m T_{S}\right)
$$

and $C^{e e}$ is the autocorrelation of the ideal MLBS

$$
C^{e e}\left(m T_{S}\right)=\sum_{i} e\left(i T_{S}\right) e\left(i T_{S}+m T_{S}\right) .
$$

The system of equations described by (11) can be solved in the frequency domain as

$$
H\left(m F_{S}, n T_{\mathrm{Rx}}\right)=\frac{\Psi^{e r}\left(m F_{S}, n T_{\mathrm{Rx}}\right)}{\Psi^{e e}\left(m F_{S}\right)},
$$


where $\Psi^{e r}$ and $\Psi^{e e}$ are one-dimensional Fourier transforms of the cross-correlation and autocorrelation function $C^{e r}$ and $C^{e e}$ in the direction of the fast time axis. The impulse response $h\left(m T_{S}, n T_{\mathrm{Rx}}\right)$ can be computed from the time variant frequency response function $H\left(m F_{s}, n T_{\mathrm{Rx}}\right)$ by onedimensional inverse Fourier transform in the direction of the fast time axis. In the case of deterministic periodical signals, when whole numbers of excitation signal periods are processed, (14) expressed in the time domain reduces to

$$
h\left(m T_{S}, n T_{\mathrm{Rx}}\right)=\operatorname{IFFT}\left[\frac{\operatorname{FFT}\left[r\left(m T_{S}, n T_{\mathrm{Rx}}\right)\right]}{\operatorname{FFT}\left[e\left(m T_{S}\right)\right]}\right],
$$

where FFT[.] is the operator of one-dimensional fast Fourier transform computed along the fast time axis. Note that due to the division in (14) and (15) both equations may be mathematically classified as an ill-posed problem which is computationally unstable around zeros in the spectrum of the excitation signal or $\Psi^{e e}$. However, the spectrum of the ideal excitation signal or its autocorrelation function does not have zeros within operational frequencies of the sounder (DC to half the clock rate). At these frequencies the spectral shape of the ideal MLBS follows a (sinc) $)^{2}$ function up to the half of its first main lobe. Therefore, the estimation of the impulse response $h\left(m T_{S}, n T_{\mathrm{Rx}}\right)$ does not face instability problems in its computation at these frequencies.

In the channel sounding and modeling applications, the knowledge of the impulse response $h\left(m T_{S}, n T_{\mathrm{Rx}}\right)$ is not sufficient. It is still influenced by the impulse response of the sounder and measurement antennas. Both of them do not belong into the characteristics of the propagation channel. In order to estimate CIRF further calibration steps must be performed. The calibration removes imperfections of the measurement device and even influence of antennas may be reduced. In Section 6, we describe a sounder calibration procedure in more details.

In many UWB sensing applications knowledge of the precise CIRF is not the main objective. Battery powered UWB sensors rather aim at low complexity signal processing which allows real-time operation with constrained power resources. In those cases, the total impulse response $h\left(m T_{S}, n T_{\mathrm{Rx}}\right)$ can be approximated by the trivial solution of the Wiener-Hopf equations which is valid for an excitation signal with white spectrum

$$
h\left(m T_{S}, n T_{\mathrm{Rx}}\right) \cong \frac{C^{e r}\left(m T_{S}, n T_{\mathrm{Rx}}\right)}{C^{e e}(0)} .
$$

This approximation does not remove the spectral shape of the ideal MLBS which follows a $(\operatorname{sinc})^{2}$ function from the estimation of the total frequency response $H\left(m F_{s}, n T_{\mathrm{Rx}}\right)$. However, it is still sufficient to evaluate performance of many sensor systems and it was even used in some spread spectrum channel sounding papers, for example, [41-43]. It is an impulse compression that cross-correlates measured signal with the ideal excitation signal

$$
h\left(m T_{S}, n T_{\mathrm{Rx}}\right) \cong \sum_{i=0}^{M-1} r\left(i T_{S}, n T_{\mathrm{Rx}}\right) e\left(m T_{S}+i T_{S}\right) .
$$

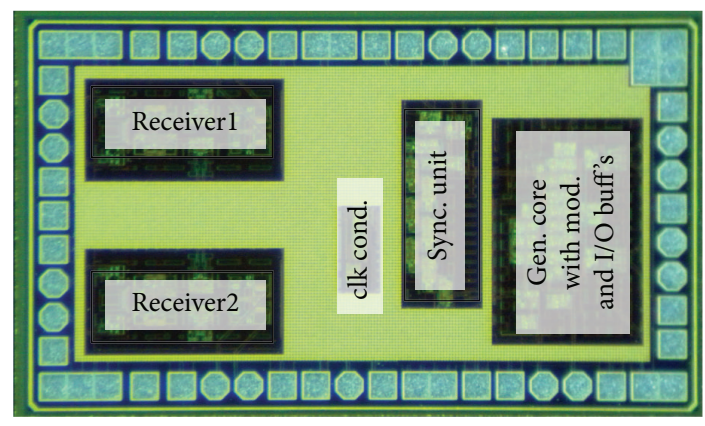

Figure 2: Single chip UWB front-end.

In the case of the binary MLBS excitation signal, this impulse compression can be done in real-time by the Fast HadamardTransform that is implemented in FPGA. The algorithm is very close to the FFT-algorithm except that it is based on a pure summing of data samples which promises very fast operation for the special hardware implementation.

\section{Channel Sounder Prototype}

The true multichannel architecture called for solid state integrated solutions since otherwise the high switching rate requirements could not be economically achieved. Therefore, the key-components of the baseband system, the MLBS generator, the binary divider, and the T\&H-circuit were manufactured in low cost, high performance $0.25 \mu \mathrm{m}$ SiGe:C BiCMOS-semiconductor technology. An example of a single chip UWB front-end is shown in Figure 2. It has a size of $2 \mathrm{~mm}^{2}$ and it integrates one MLBS generator, the binary divider, and T\&Hs for 2 receiving channels.

The shift register was realized by 12 flip-flops with the feedback structure of Fibonacci type [9]. It generates MLBS with 4095 samples. It is driven by a stable dielectric resonance oscillator (DRO) working at $f_{s}=7 \mathrm{GHz}$. In the sounder prototype we used a commercial off-the-shelf DRO from Nexyn Corporation (NXPLOS-DL). The DRO frequency determines the duration of one period of the excitation signal which is $585 \mathrm{~ns}$. That duration seems to be sufficient for anticipated indoor applications of the UWB technology. This was proven by a number of measurement campaigns [35-48].

The binary divider is responsible for providing a stable clock signal for the signal capturing and conversion which involves a wideband T\&H-stage (internally routed), a commercial video analog digital converter (ADC) (externally connected) and preprocessing such as synchronous averaging in FPGA and pulse compression in DSP. It was realized by a nine stage binary counter which is equipped by input/output buffers and supplemental synchronization flip-flop at the end of the divider chain. This removes counter timing ambiguities and features sampling clock synchronization error which lies in subpicosecond region [9].

The sampling unit works in the T\&H-mode. The actual sampling gate is embedded between a low-noise preamplifier and an output buffer of high input impedance [9]. It is driven by the sampling clock which is provided by the clock divider 
that realizes the subsampling factor of 512. This results in the sampling rate of $13.7 \mathrm{MHz}$ and allows usage of a cheap offthe-shelf ADC and yields the available measurement rate of $3345 \mathrm{CIRF} / \mathrm{s}$ per channel. Such a measurement rate is high enough to investigate fast time varying channels or to apply averaging that additionally increases the SNR of the sounder.

It must be noted that a high measurement rate may result in a huge amount of measured data that cannot be continuously stored at a normal "office" PC. With regard to our intention to build a distributed multichannel system which is capable of performing real-time measurements in different frequency bands and to continuously store data at a master PC we followed a modular concept. This resulted in the realization of UWB baseband modules, FCC up-down converter modules and $60 \mathrm{GHz}$ up-down converter modules. Basic structure of these modules is indicated for one channel configuration in Figure 1.

The baseband modules consist of UWB front-end with 1 transmitting $(\mathrm{Tx})$ and 2 receiving $(\mathrm{Rx})$ channels and a digital unit. The digital unit contains a 12 bit dual-channel ADC, one FPGA (Xilinx Spartan 3E), one DSP (Texas InstrumentsTMS 320), and a data communication interface (USB 2.0, or $100 \mathrm{MB}$ Ethernet chip). According to our experience, this approach offers a continuous measurement rate of up to $100 \mathrm{CIRF} / \mathrm{s}$ using, for example, $100 \mathrm{MB}$ Ethernet chip at the UWB baseband module and a normal "office" PC.

The core of the FCC shift register is the $0^{\circ} / 90^{\circ}$ phase switch (see Figure 1). It was realized by two microstrip delay lines of different lengths that correspond to $0^{\circ}$ and $90^{\circ}$ phase differences. The delay lines are switched by a transistor which is driven by a clock derived from the sampling clock by FPGA (see Figure 1). The mixers in the FCC converter were realized by off-the-shelf MITEQ mixers DM0412LW2.

The architecture of the $60 \mathrm{GHz}$ up-down converter represented in Figure 1 has been implemented with a singlepolarized frontend [49], with a standard WR15 waveguide transition integrated in LTCC (low temperature cofired ceramics) [50] which allows for operation of the module with different standard-interface antennas as well as for back-toback calibrations using standard devices.

The output RF power is about $0 \mathrm{dBm}$ in the baseband, minus $12 \mathrm{dBm}$ in the FCC band and $5 \mathrm{dBm}$ in the $60 \mathrm{GHz}$ band. The sensitivity of the sounder is at best described by the instantaneous dynamic range defined by

$$
\operatorname{IDR}\left(n T_{\mathrm{Rx}}\right)=10 \log \left[\frac{\max _{m T_{S}}\left(h\left(m T_{S}, n T_{\mathrm{Rx}}\right)\right)}{n_{\mathrm{avr}}}\right],
$$

where $n_{\mathrm{avr}}$ is the averaged noise floor. In all three bands it reaches about $67 \mathrm{~dB}$. More detailed discussion about the instantaneous dynamic range is given in the following section describing the sounder's calibration.

In order to efficiently use this dynamic range for signals received at different power levels UWB modules are equipped with the AGC. The AGC was realized by UWB low noise amplifiers (AVG5-03001100-50-10P-8-S from MITEQ) with the voltage controlled gain. The AGC gain is adjusted according to the power of the received signal which is estimated in the FPGA of the baseband module. Information about

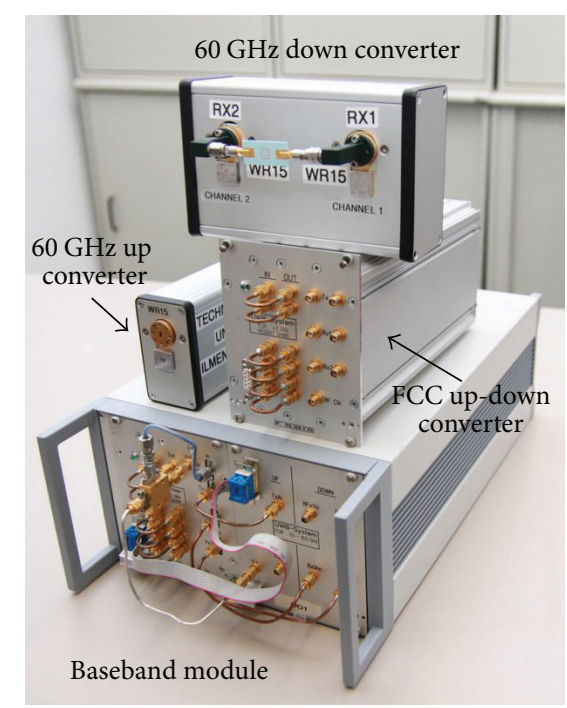

FIGURE 3: Examples of UWB baseband, FCC and $60 \mathrm{GHz}$ modules.

the AGC settings is continuously stored in the header of measured data. It is used to properly adjust power levels of recorded CIRFs and it can even be used for precise sounder calibration (see Section 6).

Figure 3 shows examples of UWB modules. 1Tx-2Rx channel sounding unit built in a 19 inch rack is illustrated at the bottom of Figure 3. It consists of the baseband module which is connected to the FCC up/down converter equipped with the AGC.

At the top of the 19 inch rack, there is a $60 \mathrm{GHz}$ downconverter and a separate baseband unit that can operate externally or in a master device. On top of it, there is a dual channel $60 \mathrm{GHz}$ down converter which is connected to a dual-polarized patch antenna. Note that this antenna is just for the use in $60 \mathrm{GHz}$ band. The channel sounding in different bands and different application scenarios requires the use of different antennas. For example, channel sounding which aims at the radio channel modeling should prefer omnidirectional antennas (such as biconical UWB antennas [51]) that support transmission and reception of channel paths from all directions (in 2D). On the other hand, channel sounding for evaluation of, for example, UWB biomedical diagnostic systems rather needs miniature human body matched directional antennas (such as Horn antennas [52]) that cover the inspected area and reduce disturbing reflections from surroundings. Antenna type, its dimensions and construction materials influence operational frequencies and radiation characteristic of the antenna. More details about the UWB antenna design can be found in [53].

Since all UWB modules are built from cost effective offthe-shelf components or customer integrated circuits as such the number of components is not an important cost factor for the overall system. This allows the construction of a true multichannel system at the receiver side and to easily emulate distributed MIMO antenna systems. The basic architecture of the proposed distributed MIMO system is shown in Figure 4. UWB modules are connected with the main control unit 


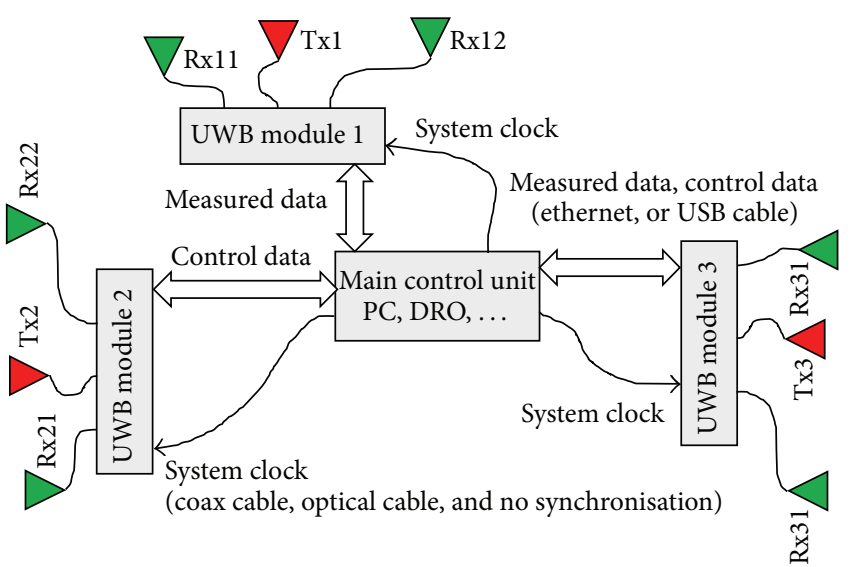

FIGURE 4: Distributed MIMO sounding system.

which saves recorded data and controls UWB modules over USB or Ethernet interface. The proposed solution of all receiving channels working in parallel provides the shortest measurement time. The transmitter modules work sequentially in time by activating only one shift register output per measurement cycle. Thus, the overall measurement time increases by the number of transmitters and does not depend on the number of receiving channels. The synchronization of the distributed system can be solved in three different ways:

(i) wired distribution of one common system clock among UWB modules by means of conventional coax cables,

(ii) distribution of the system clock as well as the data transfer by means of optical cables, and

(iii) wireless synchronization of the system clock and local data measurements at multiple control units.

The wired (coax, or optical cables) synchronization offers the most precise and stable sounding measurements. Random fluctuations of the sampling point (jitter) could be reduced down in our architecture to some tens of femtoseconds due to the balanced circuit topology and the optimized architecture of the timing system (see [9] for more details). The disadvantage of the wired synchronization is the usage of long cables in a distributed system and in the case of coax cable their influence on the propagation of EM waves and on measured data. If requirements on the synchronization are more relaxed and the sounder is not used for the channel modeling but rather for evaluation of, for example, localization systems, each UWB module (1 Tx and $2 \mathrm{Rx}$ ) can operate separately with its own system clock. In this case, the timing error that arises between 2 UWB modules is corrected offline by a software solution that performs an early-late correlation. Firstly, the measured signal is resampled with different under- and oversampling factors. Then, the resampled signal with the highest correlation is chosen to represent CIRF with the corrected timing error. Although this negatively influences performance of the sounding system and its dynamic range is reduced from $67 \mathrm{~dB}$ for a system wired synchronization to about $30 \mathrm{~dB}$,

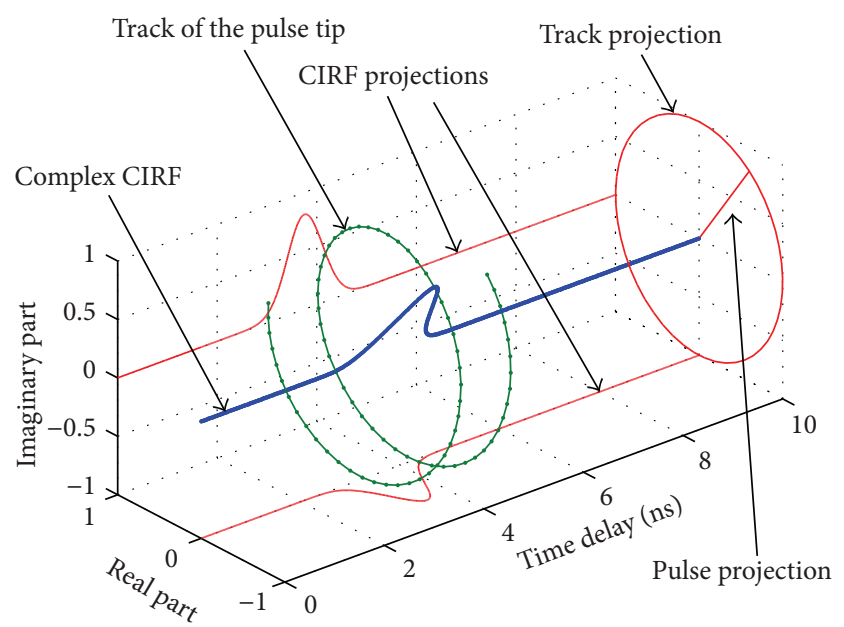

FIgURE 5: The complex CIRF of a variable delay.

the system with separately operating modules allows more realistic emulation of wireless distributed system such as sensor networks. An example of wireless tag localization by a distributed infrastructure is given in the Section 7.

\section{Sounder Calibration}

Imperfections of sounder subcomponents provoke systematic measurement errors. Main systematic errors of the sounder will be illustrated by a simple example, in which the sounder operates in a passband. Thus, it illustrates main systematic errors of the baseband part, which generates the excitation signal for the up/down converter, as well as the passband part that shifts the baseband signal to higher frequencies such as FCC and or $60 \mathrm{GHz}$ band. We assume that the transmitter is connected with the receiver via an ideal variable delay line that changes its delay during the real-time measurement. Thus, one CIRF received at a certain time is expected to contain only one complex valued pulse which propagates from the transmitter to the receiver through the delay line. An example of the ideal complex CIRF for this measurement scenario is shown in Figure 5 by the Gaussian pulse. The time location of the pulse tip as well as its twiddle angle depends on the adjusted delay time. By changing the delay time, the pulse tip moves along a screw thread track. This spiral track is illustrated in Figure 5 by the dotted line. One rotation of this track corresponds to the wavelength of the carrier signal $f_{c}$. The projection of this track on the IQplane (real and imaginary part of the CIRF) is a circle. The projection of the CIRF from Figure 5 on the IQ-plane results in a thin line as depicted in Figure 5 by "pulse projection."

However in reality, a measurement which was performed by the sounder operating in the $3.5 \mathrm{GHz}-10.5 \mathrm{GHz}$ band with connected $\mathrm{Tx}$ and $\mathrm{Rx}$ over a high quality delay line with low residual reflection produced CIRFs in which

(i) the tip of the pulse does not follow a theoretical screwed track with a circular projection on the IQplane as the delay time of the line changes. Instead, 


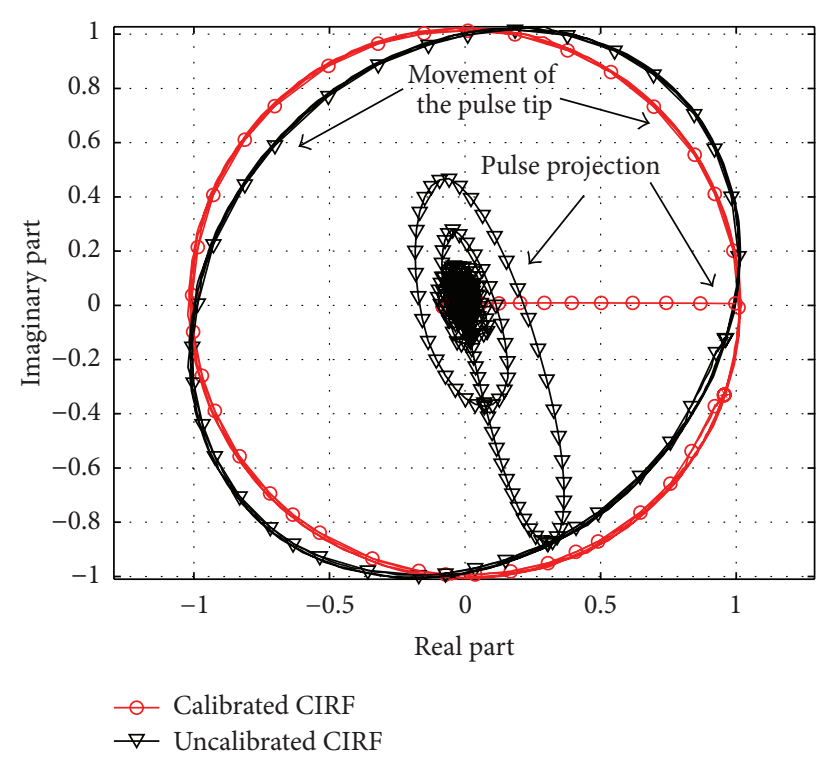

FIgURE 6: Projections of normalized CIRFs into $I$ - and Q-plain before and after calibration.

this projection has an elliptical shape as illustrated in Figure 6 by the projection of the pulse tip movement for uncalibrated CIRFs. This systematic error is caused by the IQ mismatch;

(ii) the projection of one CIRF on the IQ plane is not just one line shown in Figure 5 by "pulse projection," or in Figure 6 by the pulse projection of the calibrated CIRF (circles, solid line). It is rather looped projection as shown in Figure 6 by the pulse projection of the uncalibrated CIRF (triangles, solid line). This systematic error is caused by the impulse response of the measurement system;

(iii) instead of one pulse related to the propagation of EM waves from $\mathrm{Tx}$ to $\mathrm{Rx}$, which represents the direct wave, the measured CIRF contains additional spurious peaks as shown in Figure 7. These spurious peaks mainly arise in the CIRF due to the internal coupling between the transmitting and receiving circuitry, which is usually referred to as the crosstalk and due to the overall impulse response of the measurement system.

The way to reduce these systematic errors is a proper system calibration. The prerequisite for the successful calibration is the stable operation of the sounder over time and the availability of suitable reference objects with precisely known behavior. We propose the calibration of the sounder which operates in the passband to use 3 reference objects: $50 \mathrm{Ohm}$ impedance for a match measurement, a short transmission line for a through measurement, and the high quality delay line for IQ mismatch measurements. In the field of network analyzers, such a simplified calibration is usually referred to as the response calibration. Note, that the delay line is not necessary for the calibration of the baseband sounder. In this



Figure 7: Magnitude of an uncalibrated CIRF.

case, the received signal is real valued and there is no IQ mismatch to be corrected.

The match measurement $r_{M}\left(m T_{S}\right)$ is used for estimation of the cross-talk. In the calibration procedure, it is subtracted from uncalibrated channel measurements $r\left(m T_{S}, n T_{\mathrm{Rx}}\right)$. The through measurement $r_{T}\left(m T_{S}\right)$ is used for the estimation of the impulse (frequency) response of the system which is deconvolved from uncalibrated data with subtracted crosstalk. Thus, the calibrated CIRF $h_{c}\left(m T_{S}, n T_{\mathrm{Rx}}\right)$ can be computed from received signal $r\left(m T_{S}, n T_{\mathrm{Rx}}\right)$ as

$$
\begin{aligned}
h_{c}\left(m T_{S}, n T_{\mathrm{Rx}}\right) \\
\quad=\operatorname{IFFT}\left[\frac{\mathrm{FFT}\left[r\left(m T_{S}, n T_{\mathrm{Rx}}\right)-r_{M}\left(m T_{S}\right)\right]}{\operatorname{FFT}\left[r_{T}\left(m T_{S}\right)-r_{M}\left(m T_{S}\right)\right]}\right],
\end{aligned}
$$

where IFFT[-] is the operator of one dimensional inverse fast Fourier transform which is computed along the fast time axis. This equation is closely related to the already mentioned Wiener-Hopf method described by (15). However, apart from (15) the equation (19) may be computationally unstable around zeros in the spectrum of the through measurement $h_{T}\left(m T_{S}\right)$ with subtracted cross-talk. Therefore, application of window functions or regularized deconvolution techniques is necessary $[44,45]$.

The calibrated CIRF described by (19) is still influenced in the case of passband measurements by the IQ mismatch. The IQ mismatch measurements are used for the correction of these imperfections caused by the phase switch which enables sequential measurements of the $I$ - and Q-part of the complex valued CIRF (see Figure 1 and the related description). Although the correction of the IQ mismatch is known in the field of broadband communication systems [54-56]. To the authors' best knowledge, IQ calibration of UWB systems was not yet reported in the literature. Therefore, it is described in more details here. The imperfections of the IQ switch result in

(i) the phase difference between the $I$ - and $Q$ measurement which is not exactly $90^{\circ}$ but deviates 


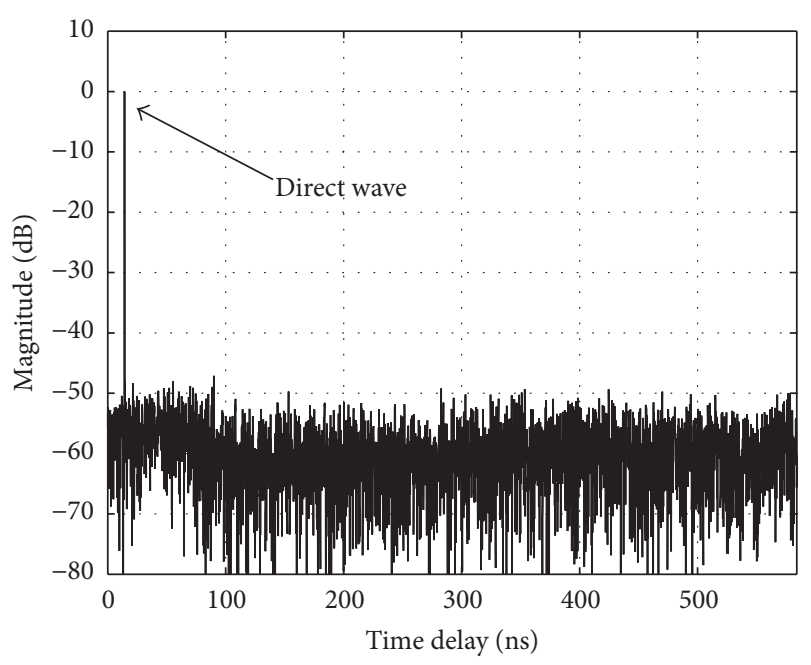

FIgURE 8: Magnitude of a calibrated CIRF.

for some degrees due to the phase error of the IQ switch and

(ii) different power levels of output signals at different states of the IQ switch, which drives the mixer (LO input).

This evokes the elliptical trace of the moving tip of the CIRF pulse as it was demonstrated in Figure 6. The ellipsis is determined by 3 parameters:

(i) A: the signal level when measuring the $I$-part,

(ii) $B$ : the signal level when measuring the $Q$-part, and

(iii) $\varphi$ : the phase error.

These parameters have to be estimated from measurements performed by the delay line at different delay times. The adjusted delays do not have to be equidistantly situated along the ellipses and they even do not need to be known. In order to completely describe the ellipsis, it is sufficient when they cover one wavelength of the carrier frequency which is related to one circulation of the pulse tip along this ellipsis. The basis for the estimation of the parameters $A, B$, and $\varphi$ are complex valued amplitudes of pulses measured at different delays. These amplitudes must be correctly extracted from measured (sampled) CIRFs. The parameters $A, B$, and $\varphi$ are estimated by means of, for example, a maximum likelihood estimator which compares extracted complex amplitudes with the model of the ellipsis. Estimated parameters are used to correct calibrated CIRF using the following equations:

$$
h_{\mathrm{CIQ}}=\frac{A+B}{2 A}\left(\operatorname{Re}\left[h_{c}\right]+i \frac{A \operatorname{Im}\left[h_{c}\right]-A B \sin \varphi \operatorname{Re}\left[h_{c}\right]}{B \cos \varphi}\right) .
$$

The result of the calibration which consists of the cross-talk removal, system impulse response calibration, and IQ mismatch correction is illustrated in Figures 6 and 8. Figure 6 shows the effect of the IQ mismatch compensation.

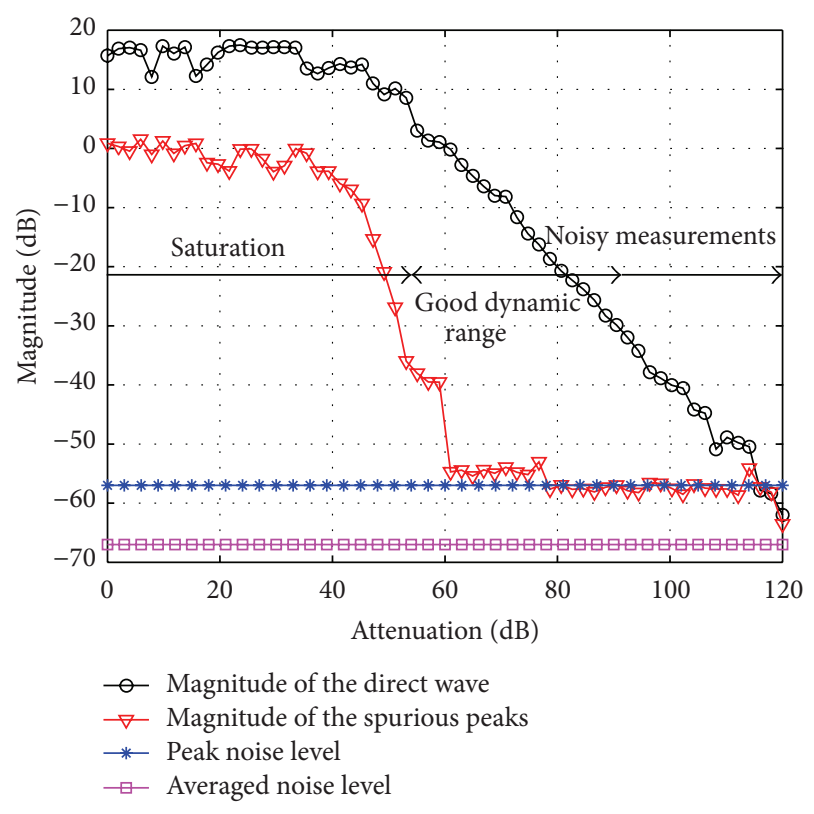

FIGURE 9: Output power versus input power.

The elliptical projection of uncalibrated measurements changes to the circular projection. Figure 8 illustrates the effect of the cross-talk and the system impulse response calibration. The spurious peaks were removed and the peak caused by the direct wave in the back to back connection was compressed in the fast-time direction (flat spectrum).

The reduction or even the removal of systematic errors increases the dynamic range of the measurement device. The dynamic range of the calibrated sounder is limited especially by the noise floor and a nonlinear system behavior. Nonlinear effects are mainly due to the saturation of amplifiers operating in the signal path. This saturation gives rise to spurious peaks that cannot be reduced by the calibration or by averaging. In order to determine the dynamic range of our sounding system, we performed the following measurement. The transmitter was connected with the receiver via an adjustable attenuator. CIRFs were estimated from data measured at different attenuations. Results of this measurement are presented in Figure 9. The figure compares the power of the direct wave to the power of maximum spurious peak. Spurious peaks are caused by either the noise of the sounder, by imperfections of the calibration, or by nonlinear effects if the input amplifiers of the sounder are oversaturated. Figure 9 shows that if the transmitted signal is attenuated by more than $115 \mathrm{~dB}$, the measured signal is hidden by the noise. If the attenuation decreases to about $60 \mathrm{~dB}$, the magnitude of the direct wave follows linear changes of the adjusted attenuation. In this region, the dynamic range is limited only by the noise floor of the sounder. If the channel attenuation is smaller than $60 \mathrm{~dB}$, the limiting factor is the nonlinear behavior of the sounder. This effect is clearly visible from about $40 \mathrm{~dB}$ attenuation. The magnitude of the direct wave does not increase any more. It is constrained especially by the sounder's amplifiers that operate in the saturation. The highest difference between the power of the direct wave and 
spurious peaks is at the attenuation of about $60 \mathrm{~dB}$. This value indicates maximum dynamic range of the sounder. It is about $67 \mathrm{~dB}$ taking into account the averaged noise level and is excellent for the system that operates in a real-time with the measurement rate of about 100 CIRF per second.

In order to efficiently use this dynamic range, the AGC adjusts the gain according to the power of the received signal. AGC increases the gain if the power of the received signal falls under a predefined level. This prohibits noisy measurements with low dynamic range (Figure 9 region at the right side). On the other side, if the signal power is too high the AGC decreases the gain and prevents nonlinear effects due to the saturation of amplifiers (Figure 9 region at the left side). Although the AGC amplifiers are capable of continuous adjustment of their gain, 16 discrete gain levels from $0 \mathrm{~dB}$ to $40 \mathrm{~dB}$ were predefined. This allows precise calibration of the sounder that is equipped with AGC. Each predefined gain level is used in additional "through" calibration measurements of AGC. These calibration measurements are used to estimate frequency responses of the AGC amplifier at the predefined gain levels. Since the information about the adjusted gain level is stored during sounding measurements the calibration of the AGC amplifier is possible by means of (18) and relevant AGC through measurement $r_{T}\left(m T_{S}\right)$.

The calibration process described above allows reduction of the systematic errors produced by the hardware of the measurement device and cables that connect it with antennas. However, it does not address calibration of the antennas and the antenna-cable connections. The proper antenna calibration is not straightforward. It is possible only with sounding measurements performed in a MIMO arrangement. MIMO CIRFs are processed by a double directional channel parameter estimator $[57,58]$ which decomposes measured CIRFs and provides angles-of-departure and angles-of-arrival for each channel path. The radiation characteristics of Tx and $\mathrm{Rx}$ antennas must be taken into account by this double directional channel estimation. A double directional channel model [59] can exploit estimated double directional channel parameters and provide a channel model with compensated antenna characteristics. Due to its complexity, the benefit of the antenna calibration is questionable since channel sounding studies are usually interested in channel statistics that incorporate application specific antennas.

\section{Chanel Sounder Application Examples}

The scope of applications of the channel sounder proposed in this paper is very wide. The baseband sounding is mainly intended for the design of through-wall radars, indoor localization, or imaging systems, and so forth. Examples of the baseband sounding can be found in $[47,48,60,61]$. The sounding in the FCC band and the $60 \mathrm{GHz}$ band is a prerequisite for the design of license free communication systems. Examples of the FCC band sounding are given, for example, in [35-46, 62].

Since this paper addresses the real-time sounding in distributed systems and since the UWB technology promises interesting perspectives for communication systems with integrated localization capabilities in indoor scenarios we

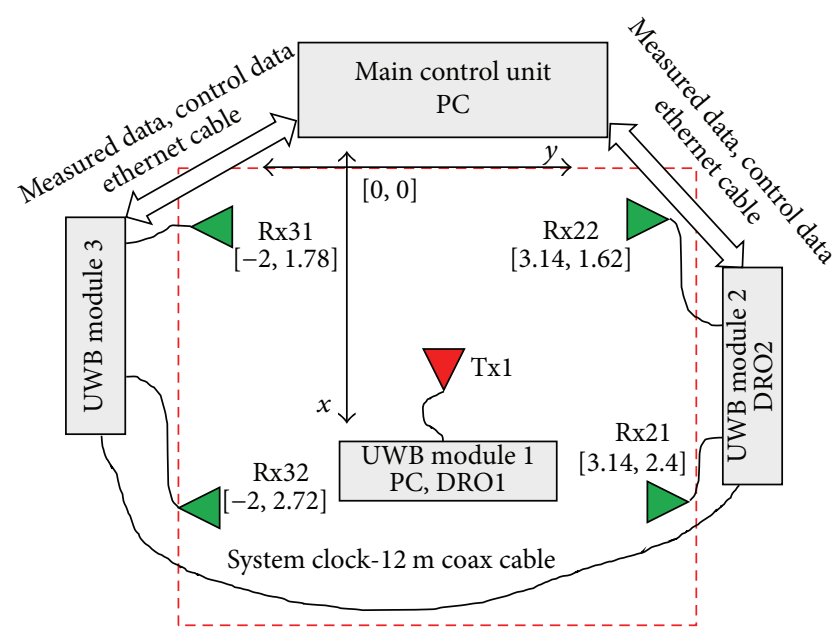

FIGURE 10: Measurement constellation.

demonstrate the localization aspect in the FCC band by the following measurement example. The goal of this sounding example is to demonstrate the localization of a moving tag in an indoor scenario where a local infrastructure is available. Note that the main objective of this example is not an analysis of the localization precision but the demonstration of the distributed sounding with the wireless synchronization between the tag and the infrastructure.

For this purpose we used three channel sounder modules that operated in the FCC band. The measurement rate was set to 50 CIRFs per second. We used omnidirectional biconical antennas. Channel sounding was performed in line-of-sight situation with the total power of the Tx module of about $-12 \mathrm{dBm}$ in a room with dimensions of $4.5 \mathrm{~m} \times 5.2 \mathrm{~m} \times$ $2.7 \mathrm{~m}$. Note that the size of the test area and the line-ofsight situation does not represent constrictions given by the channel sounder. The wireless synchronization between the tag and the infrastructure also works in non-line-of-sight situations. The ambiguity range of the sounder is given by the length of one MLBS. It is $585 \mathrm{~ns}$ and it results in the ambiguity range of about $175 \mathrm{~m}$ for the one way transmission in air. Besides the ambiguity range, the coverage area of the channel sounding system depends on the transmitted power and on properties of the propagation environment. Our tests have shown that with appropriate power amplifiers it is possible to perform measurements covering an area which is determined by the ambiguity range of the sounder. Thus, the presented application example is just a demonstration of the sounding principles in a very specific scenario.

The measurement constellation is illustrated in Figure 10. The local infrastructure was emulated by two UWB modules (2 and 3). Both UWB modules used the same system clock provided by the DRO2 $(\sim 7 \mathrm{GHz})$ which was distributed to the UWB module 3 by a $12 \mathrm{~m}$ long coax cable. The main control unit stored measured data from 4 receiving channels in parallel. UWB modules 2 and 3 were used for the reception of the signal transmitted from a moving tag. In order to perform a realistic experiment the receiving antennas were placed near walls of the measurement room. This emphasized strong multipath effects by reflections of EM waves at the walls. 


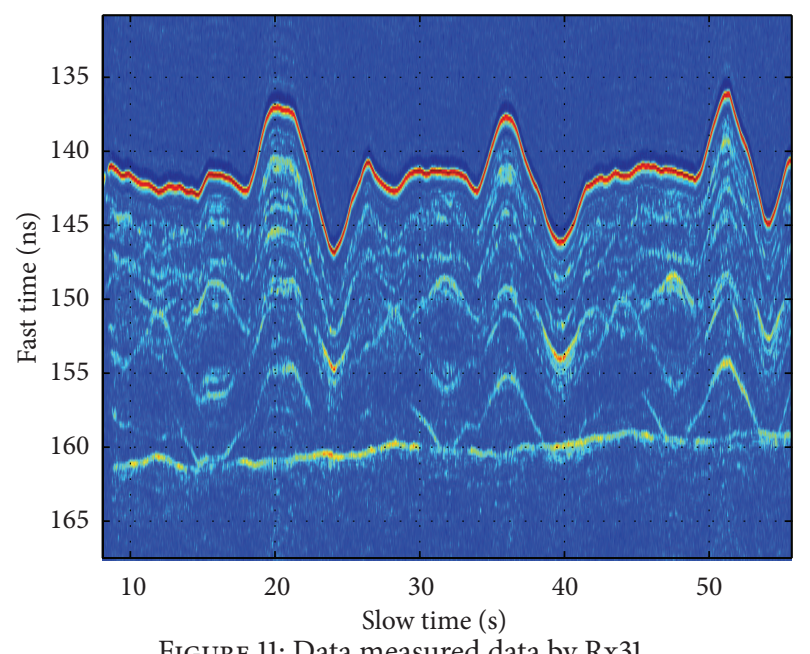

FIgure 11: Data measured data by Rx31.

The tag was emulated by the UWB module 1 . This module had its own DRO1 $(\sim 7 \mathrm{GHz})$ and was used for the transmission only. The transmitting antenna Txl was moved during the real-time measurement by a person. The person was standing under the antenna and moved it along a cross shaped track. Even the tag and the receiving modules were driven by DROs working at $7 \mathrm{GHz}$ frequencies their operation was asynchronous due to a small frequency difference between DRO1 and DRO2. However, CIRFs can be still estimated from measured data by means of an off-line signal processing which performs an early-late correlation. CIRFs estimated from data measured by $\mathrm{R} \times 31$ are shown in Figure 11. The direct wave and even the multipath effects are clearly visible in the estimated CIRFs.

Due to the missing synchronization the CIRFs can only be used for the estimation of time difference of arrivals (TDoAs). TDoAs are related to the difference of distance between the transmitting antenna and a pair of receiving antennas. TDoAs related to all combinations of receiver pairs were used in a least squares based location estimation that provided instantaneous position estimates of the moving transmitter. Figure 12 shows location estimates smoothed by an exponential averaging. Due to the handheld movement of the Tx antenna no reference track is available. However, our results indicate that a localization precision in an order of some centimeters is feasible by means of UWB technology in realistic indoor scenarios with wireless synchronization. More detailed precision analysis would require an extensive feasibility study since the precision depends not only on the quality of measured sounding data, but also on the performance of ranging, localization and tracking algorithms and on the geometry of the scenario. Our simplified feasibility study to the ranging precision of our MLBS approach was reported in [63] which showed that the excellent stability of the MLBS based UWB front-end allows ranging precision of some micrometers in a well-controlled environment.

\section{Conclusion}

The paper provides a compact description of the real-time MIMO UWB channel sounder, its design, construction,

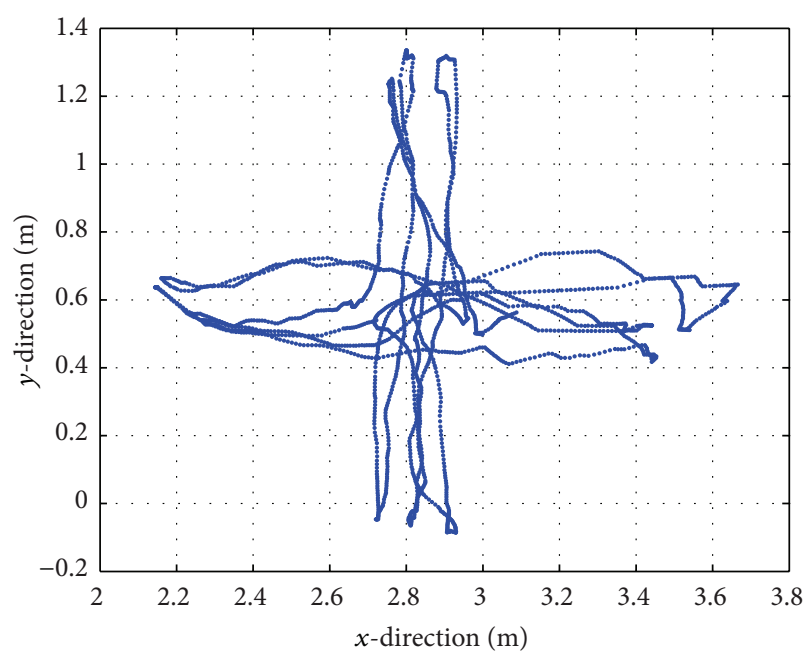

FIGURE 12: Location estimates of a moving tag.

basic parameters, calibration, and an application example for sounding in distributed systems. The architecture of the channel sounder allows continuous measurements with the data rate up to thousands of CIRFs per second in three different frequency bands. Since the measurement rate is critical for sounding of time variant channels we analyzed dependency between the measurement rate and the maximum relative radial speed of objects within the measurement scenario. For a simplified scenario, we analytically derived an expression for the measurement rate which is dependent on the object's speed and operational frequencies.

The paper described the calibration procedure of the sounder which is a prerequisite for correct channel sounding and modeling. Particularly, the IQ mismatch calibration was described in details since it is novel in the field of UWB sensors.

The modular construction of the sounder allows emulation of distributed sensor networks and offers interesting applications of the sounder. We demonstrated a novel application of distributed sounding for realistic evaluation of a UWB localization system. In this example, separated (unsynchronized) modules of the sounder emulated a tag and an infrastructure. This example showed the flexibility of the sounder. Thanks to the high degree of hardware flexibility, the construction of the sounder can be adopted to specifics of applications envisaged by the end user. The end user can predetermine a number of parameters like the number of channels, the length of measured CIRFs, the measurement rate, the operating frequencies, and the application software.

The presented sounder or its submodules were already successfully used for measurement campaigns in various environments and scenarios such as an industry and office environments aiming at the characterization and development of real time channel model [46], in homeentertainment and automotive environment aiming at the localization of tag-free persons $[62,64]$, or in public transport environment (airplane) inspecting FCC UWB and $60 \mathrm{GHz}$ transmission channel [37]. 


\section{Appendix}

\section{IQ Calibration}

Let us assume the measurement example described in the Section 6. The normalized pulse tip (Figures 5 and 6) is described in an ideal case $(A=B=1$ and $\varphi=0)$ by its $I$ and $Q$ components:

$$
I=\cos \alpha, \quad Q=\sin \alpha,
$$

where $\alpha$ is the twiddle angle which depends on the adjusted delay time in the measurement.

Due to the IQ mismatch $(\varphi \neq 0)$ and different signal levels when measuring the $I$-part and the $Q$-part $(A \neq B)$ the measured $I$ and $Q$ components are

$$
\begin{aligned}
& I_{M}=A \cos \alpha=A I, \\
& Q_{M}=B \sin (\alpha+\varphi)=B Q \cos \varphi+B I \sin \varphi,
\end{aligned}
$$

where we used (A.1) to express measured $I_{M}$ and $Q_{M}$ by the $I$ and $Q$ components of the ideal pulse tip. If the parameters $A$, $B$, and $\varphi$ are known they can be used to calibrate $I_{M}$ and $Q_{M}$ according to

$$
\begin{aligned}
& I_{C}=\frac{I_{M}}{A}, \\
& Q_{C}=\frac{Q_{M}-B I \sin \varphi}{B \cos \varphi},
\end{aligned}
$$

which is derived from (A.2) and provides calibration of the normalized impulse tip. These two equations are the basis for calibration of each complex sample of CIRF $h_{c}\left(m T_{s}, n T_{\mathrm{Rx}}\right)$

$$
\begin{aligned}
& \operatorname{Re}\left[h_{C I Q}\right]=\frac{(A+B)}{2} \frac{\operatorname{Re}\left[h_{c}\right]}{A}, \\
& \operatorname{Im}\left[h_{C I Q}\right]=\frac{(A+B)}{2} \frac{\left(\operatorname{Im}\left[h_{c}\right]-B \operatorname{Re}\left[h_{c}\right] \sin \varphi\right)}{B \cos \varphi},
\end{aligned}
$$

where we assumed that the magnitude of the IQ calibrated CIRF is equal to the mean value of $A$ and $B$ parameters. Equations (A.4) are combined and result in (20).

\section{Conflict of Interests}

The authors declare that there is no conflict of interests regarding the publication of this paper.

\section{References}

[1] M. Z. Win and R. A. Scholtz, "Impulse radio: how it works," IEEE Communications Letters, vol. 2, no. 2, pp. 36-38, 1998.

[2] Book of vision 2001; paragraph 5.4.3 New Air Interfaces. Wireless World Research Forum (WWRF) http://www.wirelessworld-research.org/.

[3] R. Zetik, J. Sachs, and R. Thomä, "UWB short range radar sensing," IEEE Instrumentation \& Measurement Magazine, vol. 9, no. 1, pp. 39-45, 2007.
[4] R. S. Thomä, D. Hampicke, A. Richter, G. Sommerkorn, and U. Trautwein, "MIMO vector channel sounder measurement for smart antenna system evaluation," European Transactions on Telecommunications, vol. 12, no. 5, pp. 427-438, 2001.

[5] R. S. Thomä, D. Hampicke, A. Richter et al., "Identification of time-variant directional mobile radio channels," IEEE Transactions on Instrumentation and Measurement, vol. 49, no. 2, pp. 357-364, 2000.

[6] Federal Communications Commission, "Part 15-radio frequency devices section 15.255: operation within the band 57.064.0 GHz," Code of Federal Regulations, FCC, 2001.

[7] "The European table of frequency allocations and utilizations covering the frequency range $9 \mathrm{kHz}$ to $275 \mathrm{GHz}$," ERC Report 25 , ERC, Lisbon, Portugal, 2002.

[8] http://www.eat.rsm.govt.nz/cms/policy-and-planning/.

[9] J. Sachs, Handbook of Ultra-Wideband Short-Range Sensing, ISSN 978-3-527-40853-5, John Wiley \& Sons, New York, NY, USA, 2012.

[10] A. F. Molisch, "Ultrawideband propagation channels-theory, measurement, and modelling," IEEE Transactions on Vehicular Technology, vol. 54, no. 5, pp. 1528-1545, 2005.

[11] J. Keignart and N. Daniele, "Subnanosecond UWB channel sounding in frequency and temporal domain," in Proceedings of the IEEE Conference on Ultra Wideband Systems and Technologies: Digest of Papers, pp. 25-30, 2002.

[12] D. Singh, H. Zhen, and R. Qiu, "UWB channel sounding and channel characteristics in rectangular metal cavity," in Proceedings of the IEEE Southeastcon, pp. 323-328, April 2008.

[13] R. Geise, I. Schmidt, M. Schack, J. Schuur, and T. Kurner, "Influence of UWB-antennas on UWB-channel-measurements in a city-liner coach," in Proceedings of the 3rd European Conference on Antennas and Propagation (EuCAP '09), pp. 3654-3657, March 2009.

[14] R. Saadane, M. Wahbi, A. Hayar, and D. Aboutajdine, "Path loss analysis based on UWB channel measurements," in Proceedings of the IEEE/ACS International Conference on Computer Systems and Applications (AICCSA '09), pp. 970-974, May 2009.

[15] B. Alavi, N. Alsindi, and K. Pahlavan, "UWB channel measurements for accurate indoor localization," in Proceedings of the Military Communications Conference (MILCOM '06), pp. 1-7, October 2006.

[16] S. Bories, A. Sibille, and C. Roblin, "UWB indoor channel measurements study," in Proceedings of the IEEE International Workshop on Antenna Technology: Small Antennas and Novel Metamaterials (IWAT '05), pp. 466-469, March 2005.

[17] A. Dezfooliyan and A. M. Weiner, "Evaluation of time domain propagation measurements of UWB systems using spread spectrum channel sounding," IEEE Transactions on Antennas and Propagation, vol. 60, no. 10, pp. 4855-4865, 2012.

[18] D. Sugizaki, N. Iwakiri, and T. Kobayashi, "Ultra-wideband spatio-temporal channel sounding with use of an OFDM signal in an indoor environment," PIERS Online, vol. 7, no. 2, pp. 113116, 2011.

[19] W. Ciccognani, A. Durantini, and D. Cassioli, "Time domain propagation measurements of the UWB indoor channel using PN-sequence in the FCC-compliant band 3.6-6 GHz," IEEE Transactions on Antennas and Propagation, vol. 53, no. 4, pp. $1542-1549,2005$.

[20] R. J. Pirkl, A sliding correlator channel sounder for UWB measurements [M.S. Thesis], Georgia Institute of Technology, August 2007. 
[21] http://www.euwb.eu/.

[22] J. Sachs, R. Thomä, U. Schultheiss, R. Zetik, J. Dvoracek, and M. Wolf, "Real-time ultra-wideband channel sounder," in Proceedings of 27th General Assembly URSI, Maastricht, The Netherlands, August 2002.

[23] M. Kmec, J. Sachs, P. Peyerl, P. Rauschenbach, R. Thomä, and R. Zetik, "A novel ultra-wideband real-time MIMO channel sounder architecture," in Proceedings of the 28th General Assembly of URSI, New Delhi, India, October 2005.

[24] Medav Rusk Mimo Channel Sounder Manual.

[25] Elektrobit PROPsound MIMO Channel Sounder Manual.

[26] H.-K. Chung, N. Vloeberghs, H. K. Kwon, S. J. Lee, and K.-C. Lee, "MIMO channel sounder implementation and effects of sounder impairment on statistics of multipath delay spread," in Proceedings of the IEEE 62nd Vehicular Technology Conference (VTC '05), vol. 1, pp. 349-353, September 2005.

[27] B. T. Maharaj, L. P. Linde, J. W. Wallace, and M. A. Jensen, "A cost-effective wideband MIMO channel sounder and initial colocated $2.4 \mathrm{GHz}$ and $5.2 \mathrm{GHz}$ measurements," in Proceedings of the IEEE International Conference on Acoustics, Speech, and Signal Processing (ICASSP '05), pp. III981-III984, March 2005.

[28] V. Kolmonen, P. Almers, J. Salmi et al., "A dynamic duallink wideband MIMO channel sounder for $5.3 \mathrm{GHz}$," IEEE Transactions on Instrumentation and Measurement, vol. 59, no. 4, pp. 873-883, 2010.

[29] G. J. M. Janssen and J. A. M. Vriens, "High resolution coherent radio channel measurements using direct sequence spread spectrum modulation," in Proceedings of the 6th Mediterranean Electrotechnical Conference (Melecon '91), vol. 1, pp. 720-727, LJubljana, Slovenia, May 1991.

[30] J. Sachs, P. Peyerl, and R. Zetik, "Stimulation of UWB-sensors: pulse or maximum sequence?" in Proceedings of the International Workshop on UWB Systems, p. 5, Oulu, Finland, June 2003.

[31] J. Sachs, P. Peyerl, and M. Rossberg, "A new UWB-principle for sensor-array application," in Proceedings of the IEEE 16th Instrumentation and Measurement Technology Conference (IMTC'99), vol. 3, pp. 1390-1395, Venice, Italy, May 1999.

[32] J. Sachs, P. Peyerl, F. Tkac, and M. Kmec, "Digital ultrawideband-sensor electronics integrated in SiGe-technology," in Proceedings of the 32nd European Microwave Conference (EuMC '02), pp. 539-542, Milan, Italy, September 2002.

[33] J. Sachs, M. Kmec, and R. Zetik, "Ultra wideband radar assembly kit," in Proceedings of the IEEE International Geoscience and Remote Sensing Symposium (IGARSS '05), pp. 372-375, Soul, Republic of Korea, August 2005.

[34] J. Sachs, M. Kmec, P. Payerl, P. Rauschenbach, and R. Zetik, "MSCW-radar-a novel ultra wideband radar principle," in Proceedings of the International Radar Symposium, Berlin, Germany, September 2005.

[35] A. P. Garcia Ariza, W. Kotterman, R. Zetik et al., "60 GHzultrawideband real-time multi-antenna channel sounding for multi giga-bit/s access," in Proceeding of the 72nd IEEE Vehicular Technology Conference Fall (VTC '10), pp. 1-6, Ottawa, Canada, September 2010.

[36] A. P. Garcia, W. Kotterman, U. Trautwein, D. Brückner, J. Kunisch, and R. S. Thomä, "60 GHz Time-Variant Shadowing Characterization within an Airbus 340," in Proceedings of the 4th European Conference on Antennas and Propagation (EuCAP '10), Barcelona, Spain, April 2010.
[37] A. P. Garcia, W. Kotterman, R. Thomä et al., " $60 \mathrm{GHz}$ in-cabin real-time channel sounding," in Proceedings of the 3rd International Workshop on Broadband MIMO Channel Measurement and Modeling (IWonCMM '09), Xian, China, 2009.

[38] A. P. G. Ariza, R. Müller, R. Stephan et al., " $60 \mathrm{GHz}$ polarimetric MIMO sensing: architectures and technology," in Proceedings of the 6th European Conference on Antennas and Propagation (EUCAP '12), pp. 2578-2582, Prague, Czech Republic, March 2012.

[39] A. P. G. Ariza, R. Müller, F. Wollenschläger et al., “ $60 \mathrm{GHz}$ ultrawideband polarimetric MIMO sensing for wireless multigigabit and radar," IEEE Transactions on Antennas and Propagation, vol. 61, no. 4, pp. 1631-1641, 2013.

[40] J. S. Bendat and A. G. Piersol, Engineering Applications of Correlation and Spectral Analysis, John Wiley \& Sons, 1980.

[41] G. J. M. Janssen and J. A. M. Vriens, "High resolution coherent radio channel measurements using direct sequence spread spectrum modulation," in Proceedings of the 6th Mediterranean Electrotechnical Conference (Melecon '91), vol. 1, pp. 720-727, May 1991.

[42] A. Durantini, W. Ciccognani, and D. Cassioli, "UWB propagation measurements by $\mathrm{PN}$-sequence channel sounding," in Proceedings of the IEEE International Conference on Communications, pp. 3414-3418, Paris, France, June 2004.

[43] D. Cassioli and A. Durantini, "Measurements, modeling and simulations of the UWB propagation channel based on directsequence channel sounding," Wireless Communications and Mobile Computing, vol. 5, no. 5, pp. 513-523, 2005.

[44] T. G. Savelyev and M. Sato, "Comparative analysis of UWB deconvolution and feature-extraction algorithms for GPR landmine detection," in Detection and Remediation Technologies for Mines and Minelike Targets IX, vol. 5415 of Proceedings of SPIE, September 2004.

[45] T. G. Savelyev, L. van Kempen, and H. Sahli, "Deconvolution techniques," in Ground Penetrating Radar, D. Daniels, Ed., vol. 15, pp. 298-310, IEE Radar, Sonar, Navigation and Avionics Series, Bodmin, UK, 2nd edition, 2004.

[46] J. Pamp, J. Kunisch, and R. Zetik, “Time-variant ultra-wideband radio channel measurements in industrial environment," in Proceedings of the ICT-MobileSummit, Stockholm, Sweden, 2008.

[47] R. Zetik, J. Sachs, and R. S. Thomä, "Distributed UWB MIMO sounding for evaluation of cooperative localization principles in sensor networks," in Proceedings of the 14th European Signal Processing Conference (EUSIPCO '06), pp. 4-8, Italy, September 2006.

[48] R. Zetik, J. Sachs, and R. Thomä, "Imaging of propagation environment by UWB channel sounding," in Proceedings of the 28th General Assembly of URSI, New Delhi, India, October 2005.

[49] R. Müller, F. Wollenschläger, A. Schulz et al., " 60 GHz ultrawideband front-ends with gain control, phase shifter, and wave guide transition in LTCC technology," in Proceedings of the 6th European Conference on Antennas and Propagation (EuCAP '12), pp. 3255-3259, March 2012.

[50] F. Wollenschläger, R. Müller, and R. Stephan, "A wideband $60 \mathrm{GHz}$ differential stripline-to-waveguide transition for antenna measurements in low-temperature co-fired ceramics technology," in Proceedings of the 6th European Conference on Antennas and Propagation (EUCAP '12), pp. 3537-3541, Prague, Czech Republic, March 2012.

[51] H. Ghannoum, S. Bories, C. Roblin, and A. Sibille, "Biconical antennas for intrinsic characterization of the UWB channel," 
in Proceeding of the IEEE International Workshop on Antenna Technology: Small Antennas and Novel Metamaterials (IWAT '05), pp. 101-104, March 2005.

[52] F. S. di Clemente, R. Stephan, U. Schwarz, and M. A. Hein, "Miniature body-matched double-ridged horn antennas for biomedical UWB imaging," in Proceedings of the IEEE-APS Topical Conference on Antennas and Propagation in Wireless Communications (APWC '12), pp. 574-577, Cape Town, South Africa, September 2012.

[53] W. Wiesbeck, G. Adamiuk, and C. Sturm, "Basic properties and design principles of UWB antennas," Proceedings of the IEEE, vol. 97, no. 2, pp. 372-385, 2009.

[54] P. Rykaczewski, M. Valkama, and M. Renfors, "On the connection of I/Q imbalance and channel equalization in directconversion transceivers," IEEE Transactions on Vehicular Technology, vol. 57, no. 3, pp. 1630-1636, 2008.

[55] C. F. Gu, C. L. Law, and W. Wu, "Time domain IQ imbalance compensation for wideband wireless systems," IEEE Communications Letters, vol. 14, no. 6, pp. 539-541, 2010.

[56] C. R. Rojas, P. Zetterberg, and P. Handel, "Transceiver inphase/quadrature imbalance, ellipse fitting, and the universal software radio peripheral," IEEE Transactions on Instrumentation and Measurement, vol. 60, no. 11, pp. 3629-3639, 2011.

[57] K. Haneda and J. Takada, "An application of SAGE algorithm for UWB propagation channel estimation," in Proceedings of the IEEE Conference on Ultra Wideband Systems and Technologies, pp. 483-487, November 2003.

[58] K. Haneda, J. Takada, and T. Kobayashi, "A parametric UWB propagation channel estimation and its performance validation in an anechoic chamber," IEEE Transactions on Microwave Theory and Techniques, vol. 54, no. 4, pp. 1802-1811, 2006.

[59] K. Haneda, J. Takada, and T. Kobayashi, "Applicability of UWB double directional propagation modeling for evaluating UWB transmission performance," in Proceedings of the IEEE 65th Vehicular Technology Conference (VTC '07), pp. 377-381, April 2007.

[60] J. Sachs, R. Zetik, P. Peyerl, and J. Friedrich, "Autonomous orientation by ultra wideband sounding," in Proceedings of the 9th International Conference on Electromagnetics in Advanced Applications (ICEAA '05), pp. 12-16, Torino, Italy, September 2005.

[61] R. Zetik, S. Crabbe, J. Krajnak, P. Peyerl, J. Sachs, and R. Thomä, "Detection and localization of persons behind obstacles using M-sequence through-the-wall radar," in Proceedings of the SPIE Defense and Security Symposium, Orlando, Fla, USA, April 2006.

[62] R. Zetik, G. Shen, and R. Thomä, "Evaluation of passive UWB localization system for home-entertainment application by the real-time UWB channel sounder," in International Conference on Indoor Positioning and Indoor Navigation, Zürich, Switzerland, September 2010.

[63] R. Zetik, J. Sachs, and P. Peyerl, "UWB radar: Distance and positioning measurements," in Proceedings of the 8th International Conference on Electromagnetics in Advanced Applications (ICEAA '03), pp. 737-740, Torino, Italy, September 2003.

[64] R. Zetik and R. Thomä, "UWB measurements and data analysis in automotive scenarios," in Proceedings of the 5th European Conference on Antennas and Propagation (EUCAP '11), pp. 3065-3069, Rome, Italy, April 2011. 

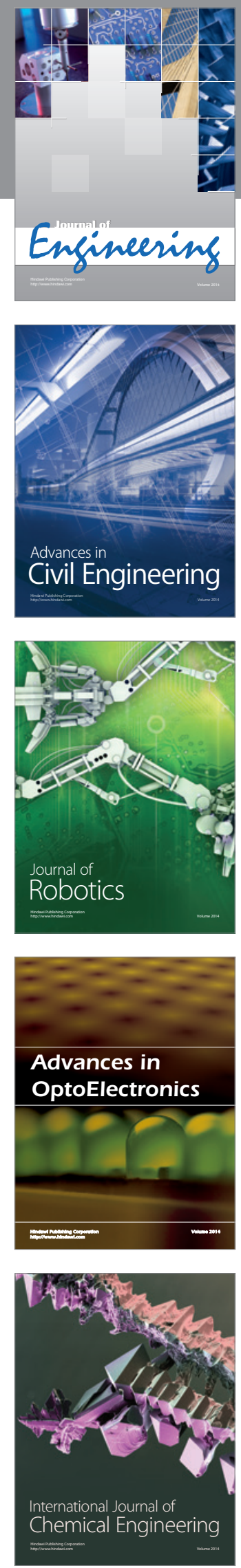

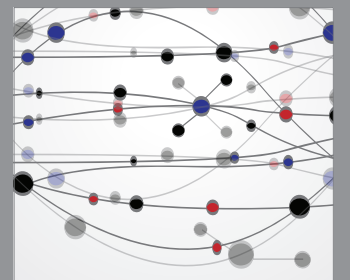

The Scientific World Journal
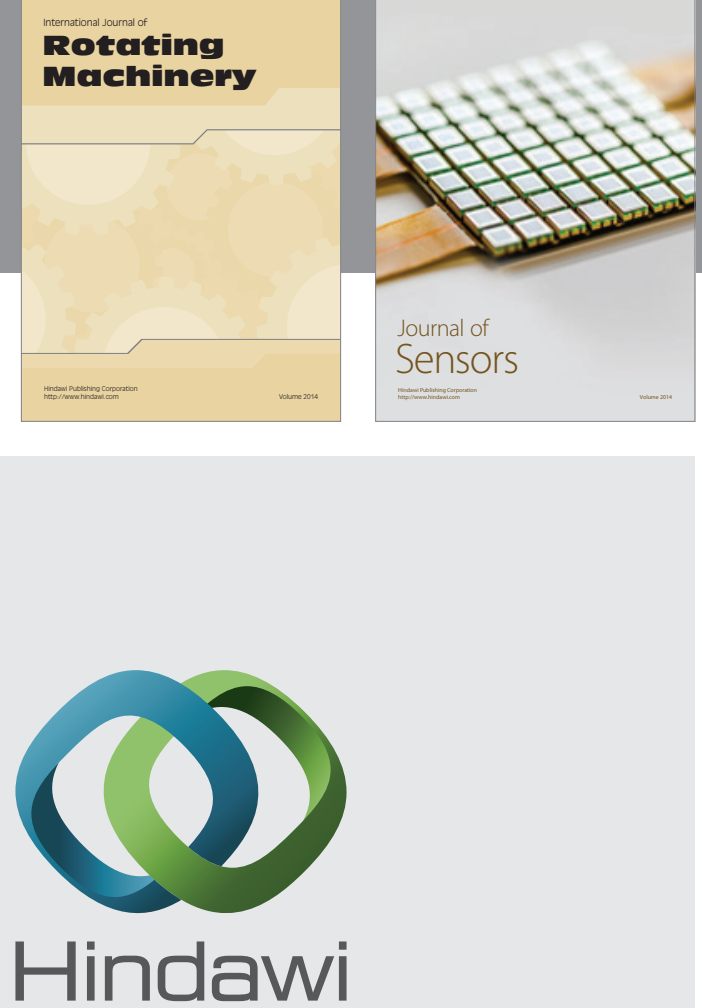

Submit your manuscripts at http://www.hindawi.com
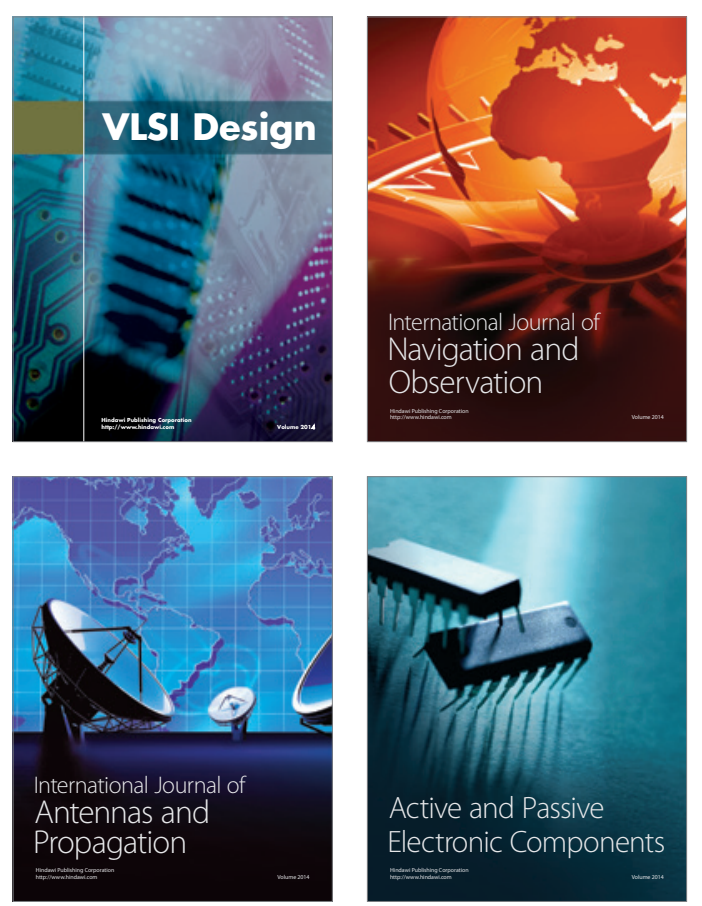
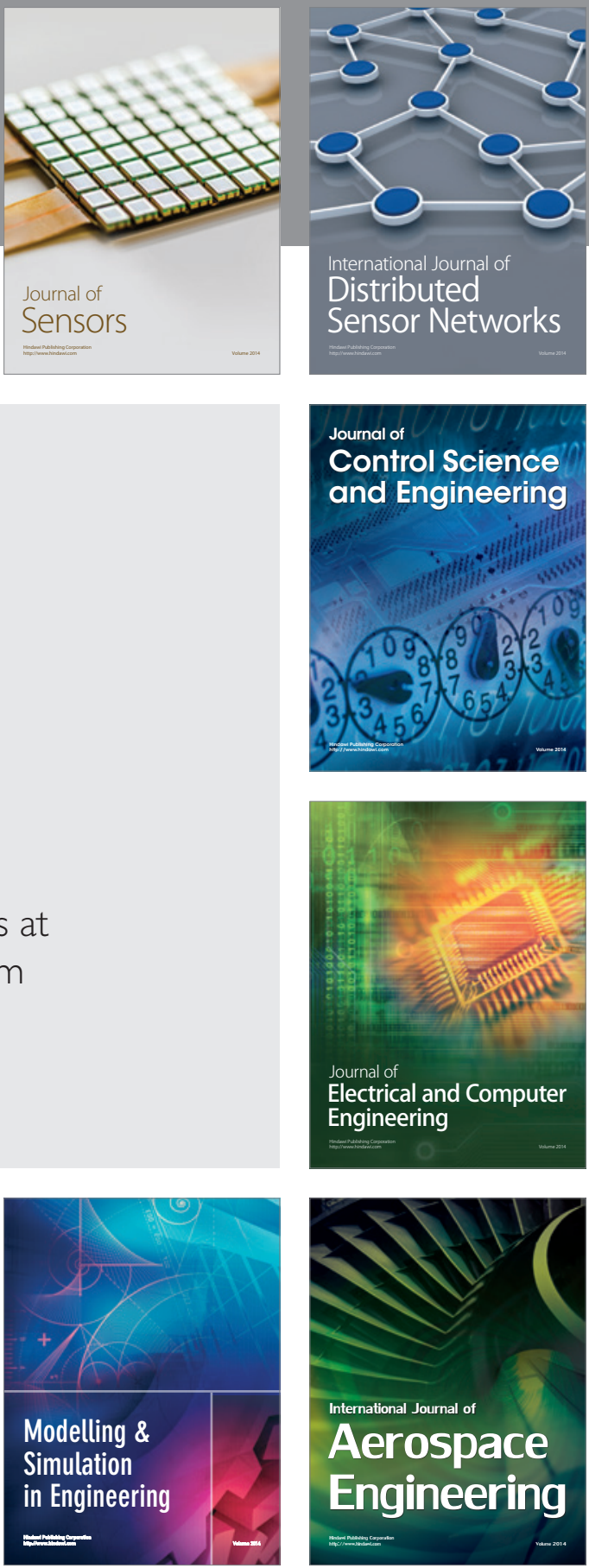

Journal of

Control Science

and Engineering
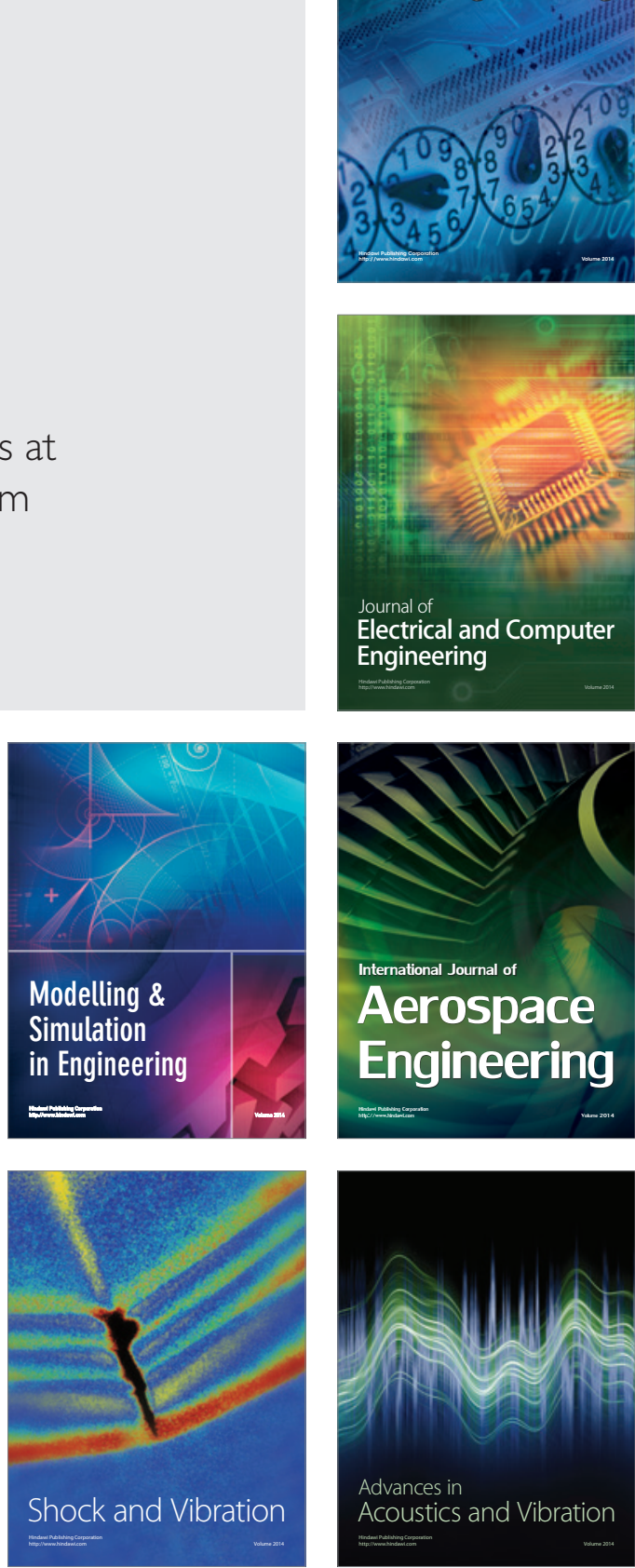